\title{
Analytical Method for Evaluation of Coupled Responses of a Multidirectionally Loaded Pile-Raft Foundation Induced by Tunnelling in Layered Soils
}

\author{
Linlong $\mathrm{Mu}^{1,2}$ and Maosong Huang ${ }^{1,2}$ \\ ${ }^{1}$ Department of Geotechnical Engineering, Tongji University, Shanghai 200092, China \\ ${ }^{2}$ Key Laboratory of Geotechnical and Underground Engineering of Ministry of Education, Tongji University, Shanghai 200092, China \\ Correspondence should be addressed to Linlong Mu; mulinlong@tongji.edu.cn
}

Received 8 April 2015; Accepted 27 May 2015

Academic Editor: Reza Jazar

Copyright ( $\odot 2015$ L. Mu and M. Huang. This is an open access article distributed under the Creative Commons Attribution License, which permits unrestricted use, distribution, and reproduction in any medium, provided the original work is properly cited.

\begin{abstract}
The tunnelling effect on a pile-raft foundation is usually studied in either the horizontal or vertical direction separately, while, in practice, the responses of a pile-raft foundation induced by tunnelling in the horizontal and vertical directions occur simultaneously. Typically, a pile-raft foundation is usually loaded by vertical and horizontal loads and moments when a tunnel is constructed. Since little effort has been conducted to evaluate the coupled responses of multidirectionally loaded pile-raft foundations subjected to tunnelling, a modified two-stage method is proposed in this paper to evaluate the coupled responses of a multidirectionally loaded pile-raft foundation in layered soil. After careful verification of the method, a parametric study was carried out to evaluate whether it is necessary to consider the influences of tunnelling on a loaded pile-raft foundation from the design stages. Our study showed that it is more necessary to consider the influence of tunnelling on a pile-raft foundation when the working load on the pile-raft foundation is small. When there was only a vertical load working on the raft, the horizontal deformation and the rotation of the raft, as well as any horizontal deformation and moment along the piles, were controlled by tunnelling.
\end{abstract}

\section{Introduction}

In order to meet a growing population's demands for space, a lot of underground space has been developed. In urban areas, where many high buildings are supported by pileraft foundations, which are recognized as one of the most economical foundation systems, addressing the effect of underground development on these foundations is a key area of interest. In recent years, numerous subway tunnels have been constructed in cities, with many of them being constructed near existing pile-supported structures. In the interest of maintaining safety of existing buildings, estimating the impact of tunnelling on existing pile-raft foundations has become a key ingredient of tunnel designs.

Plenty of researchers have carried out studies of the tunnelling effect on adjacent piles through experimental methods [1-4], the finite-element method (FEM) [5-7], and analytical approaches [8-12]. The two-stage method was proved to be the procedure that is most likely to be used in practice to calculate pile responses induced by tunnelling $[8,10-12]$. Based on analytical solutions for a multilayered soil system, some authors extended the two-stage method from a homogeneous soil system to a layered soil system $[13,14]$. Since then, most investigations concerned pile responses in the vertical or horizontal directions separately, while in practice vertical and horizontal responses occur simultaneously. As such, significant coupling effects of vertical and horizontal responses had previously been observed in a few papers [1518]. However, none of these results considered the combined response of a pile-raft foundation under "passive loads."

In addition, the existing pile-raft foundations were always subjected to a working load; however the aforementioned methods tended to neglect the coupling effect between working and passive loads induced by tunnelling on a pileraft foundation. However, according to some experimental studies [19], the influence of the working load is not 
negligible. Reference [20] presented a method to calculate the response of loaded pile groups induced by tunnelling that considered the influence of working loads; however raft-soil interaction, pile-soil surface interaction, and the coupled effect of vertical and horizontal responses were not considered in their method, where only the vertical working load was considered. However, pile-raft foundations are always subjected to horizontal loads, either a moment or load combined with a vertical load. A limited study regarding pileraft foundations subjected to combined loads was provided by [21], which presented an analytical method to calculate the responses of a pile-raft foundation under a combined load. No investigation has yet been conducted, however, to investigate the response of a multidirectionally loaded pileraft foundation subjected to tunnelling. Thus, a practical method is urgently needed to evaluate the coupled responses of a pile-raft foundation induced by tunnelling that considers the influence of multidirectional working loads.

Considering the aforementioned problems associated with the existing two-stage method, a modified two-stage method is proposed in this paper. First, an empirical method is employed to calculate free-field soil movement in the absence of existence of piles. Secondly, we impose free-field soil deformations on the piles to calculate the responses of a pile-raft foundation by considering the coupled effect of vertical and horizontal responses and the influences of working loads on the raft.

\section{Free-Field Soil Movement Induced by Tunnelling}

Many approaches, including analytical methods [22, 23], FEM [24], and empirical methods [25-27], have been presented to evaluate the free-field soil deformation induced by tunnelling. The analytical method proposed by [22] is widely recognized as one of the most important solutions for the problem $[23,27,28]$. And the empirical method proposed by [27] based on the analytical method [22] is proved to be the most useful method employed in practice $[11,13,14,20]$. Thus, the closed form of the empirical solution of free-field soil deformation induced by tunnelling is employed in this study for its easy application in layered soils:

$$
\begin{aligned}
u_{s} & =-\varepsilon_{0} R^{2} x\left\{\frac{1}{x^{2}+(z-H)^{2}}+\frac{3-4 v}{x^{2}+(z+H)^{2}}\right. \\
& \left.-\frac{4 z(z+H)}{x^{2}+(z+H)^{2}}\right\} \cdot e^{-\left[-1.38 x^{2} /(H+R)^{2}+0.69 z^{2} / H^{2}\right]}, \\
w_{s} & =\varepsilon_{0} R^{2}\left\{-\frac{z-H}{x^{2}+(z-H)^{2}}+\frac{(3-4 v)(z+H)}{x^{2}+(z+H)^{2}}\right. \\
& \left.-\frac{2 z\left[x^{2}-(z+H)^{2}\right]}{x^{2}+(z+H)^{2}}\right\} \cdot e^{-\left[-1.38 x^{2} /(H+R)^{2}+0.69 z^{2} / H^{2}\right]},
\end{aligned}
$$

where $u_{s}$ is the horizontal movement of the free-field soil; $w_{s}$ is the vertical movement of the free-field soil; $R$ is the tunnel radius; $\varepsilon_{0}$ is the average ground-loss ratio which is

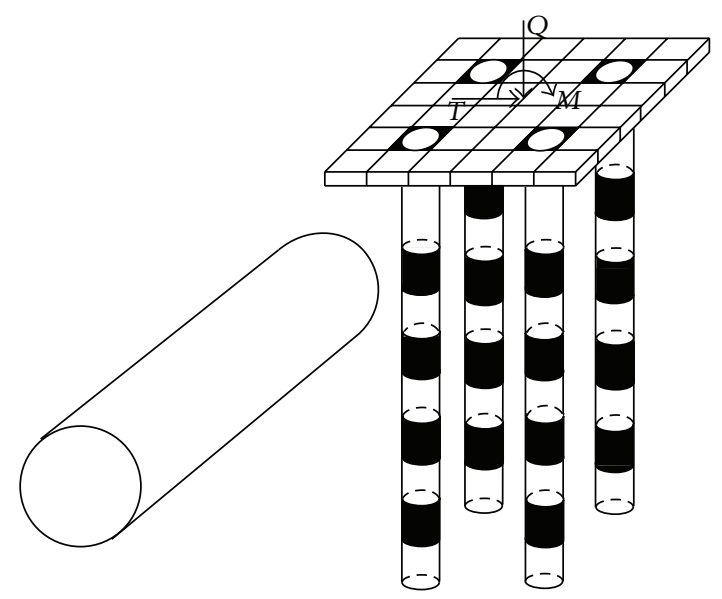

FIGURE 1: Sketch of a loaded pile-raft foundation adjacent to a tunnel.

defined as a percentage of the ratio of the surface settlement through volume and the tunnel volume per unit length; $x$ is the horizontal distance from the tunnel centerline; $z$ is the depth below the surface; $H$ is the depth of tunnel spring line; and $v$ is the soil Poisson's ratio.

\section{Analytical Method for Pile-Rafts}

The typical problem of a tunnel that is constructed adjacent to an existing pile-raft foundation, which is then subjected to a vertical load, a horizontal load, and moment from the superstructure, is shown in Figure 1. In order to evaluate the responses of the pile-raft foundation that is subjected to both active and passive loads induced by tunnelling, this section is organized as follows. In Section 3.1, a method for calculating the responses of a single pile induced by both active and passive loads in layered soils is established. In Section 3.2, the method is extended to a pile group, while, in Section 3.3, the pile-soil surface interaction and soil-soil interaction at the surface are defined. In Section 3.4, raft constraints are considered in order to establish a method for a pile-raft foundation that is subjected to both active and passive loads.

3.1. An Analytical Method for a Single Pile. Assuming that slip and separation does not occur between the pile and the soil, the schematic diagram of a loaded single pile subjected to soil movement induced by tunnelling is shown in Figure 2. Through the finite-difference method, the vertical and horizontal soil equilibrium equations can be expressed as follows:

$$
\begin{aligned}
\mathbf{w}_{p}^{0}-\mathbf{w}_{s}^{0} & =\mathbf{I}_{s V}^{0} \mathbf{q}^{0}, \\
\mathbf{u}_{p}^{0}-\mathbf{u}_{s}^{0} & =\mathbf{I}_{s H}^{0} \mathbf{p}^{0},
\end{aligned}
$$

where $\mathbf{w}_{p}^{0}$ is the vertical nodal deformation vector of the pile; $\mathbf{w}_{s}^{0}$ is the vector of the vertical free-field soil deformation induced by tunnelling; $\mathbf{I}_{s V}^{0}$ is the vertical soil flexibility matrix, which can be found in [13] for a layered soil system; $\mathbf{q}_{0}$ is 


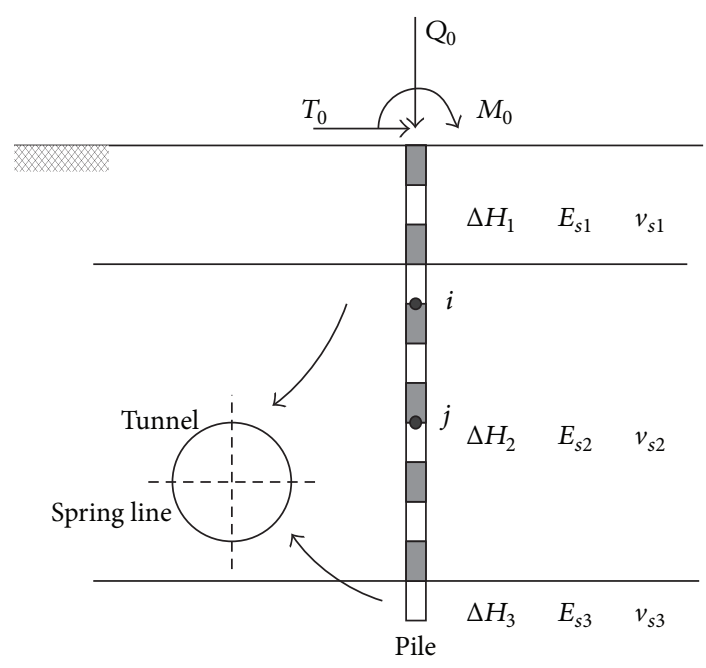

Figure 2: The problem of a single pile.

the vertical nodal soil resistance force vector of the pile; $\mathbf{u}_{p}^{0}$ is the horizontal nodal displacement vector of the pile; $\mathbf{u}_{s}^{0}$ is the vector of the horizontal free-field soil deformation induced by tunnelling; $\mathbf{I}_{s H}^{0}$ is the horizontal soil flexibility matrix, which can be found in [14] for a layered soil system; and $\mathbf{p}_{0}$ is the horizontal nodal soil resistance force vector of the pile.

The pile equilibrium equations can be expressed in matrix form as follows:

$$
\begin{gathered}
\mathbf{q}^{0}-\frac{d_{0} \cdot E_{p 0} n^{2}}{4 L_{0}^{2}} \mathbf{I}_{p V}^{0} \mathbf{w}_{p}^{0}=\mathbf{Y}_{V}^{0}, \\
\mathbf{p}^{0}-\frac{E_{p 0} I_{p 0} n^{4}}{L^{4}} \mathbf{I}_{p H}^{0} \mathbf{u}_{p}^{0}=\mathbf{Y}_{H}^{0},
\end{gathered}
$$

where $d_{0}$ is the diameter of the pile; $E_{p 0}$ is the elastic modulus of the pile; $L_{0}$ is the length of the pile; $n_{0}$ is the total number of elements along the pile; $I_{p 0}$ is the moment of inertia of the pile; $\mathbf{I}_{p V}^{0}$ is the vertical pile flexibility matrix of the pile; $\mathbf{I}_{p H}^{0}$ is the horizontal pile flexibility matrix of the pile; and $\mathbf{Y}_{V}^{0}=$ $\left\{2 Q_{0} n_{0} / \pi d_{0} L_{0} \quad 0 \quad \cdots \quad c c 0\right\}, \mathbf{Y}_{H}^{0}=\left\{\left(2 L_{0} n_{0} T_{0}+2 n_{0}^{2} M_{0}\right) /\right.$ $\left.\begin{array}{lllll}L_{0}^{2} & -n_{0}^{2} M_{0} / L_{0}^{2} & 0 & \cdots & 0\end{array}\right\}$.

From (3) and (4), the pile-soil system equilibrium equations can be written as

$$
\begin{aligned}
& \left(\mathbf{I}^{0}-\frac{E_{p 0} d_{0} n_{0}^{2}}{4 L_{0}^{2}} \mathbf{I}_{p V}^{0} \mathbf{I}_{s V}^{0}\right) \mathbf{q}^{0}=\frac{d_{0} E_{p 0} n^{2}}{4 L_{0}^{2}} \mathbf{I}_{p V}^{0} \mathbf{w}_{s}^{0}+\mathbf{Y}_{V}^{0} \\
& \left(\mathbf{I}^{0}-\frac{E_{p 0} I_{p 0} n_{0}^{4}}{L_{0}^{4}} \mathbf{I}_{p H}^{0} \mathbf{I}_{s H}^{0}\right) \mathbf{p}^{0}=\frac{E_{p 0} I_{p 0} n^{4}}{L_{0}^{4}} \mathbf{I}_{p L}^{0} \mathbf{u}_{s}^{0}+\mathbf{Y}_{H}^{0} .
\end{aligned}
$$

3.2. The Pile Group Effect. Adopting the finite-difference method, the vertical and horizontal equilibrium equations for

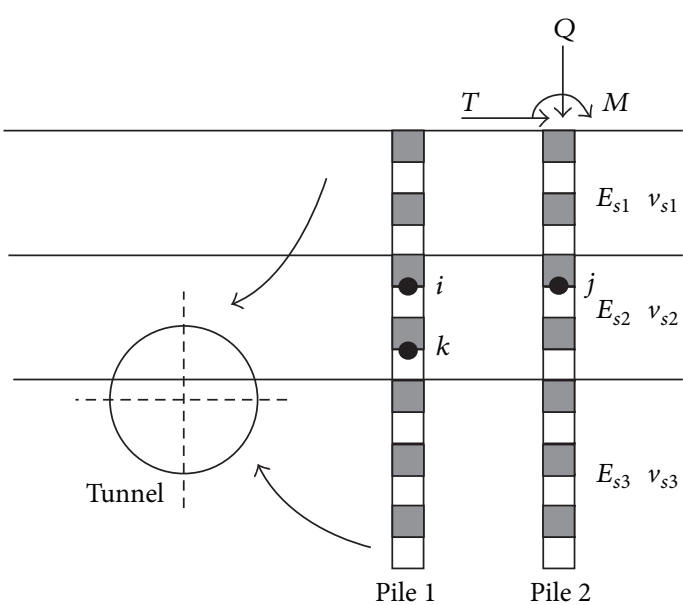

Figure 3: Two pile-tunnel systems.

the two pile-tunnel systems shown in Figure 3 can be written as follows:

$$
\begin{aligned}
& {\left[\begin{array}{cc}
\mathbf{I}^{1}-a_{1} \mathbf{I}_{p V}^{1} \mathbf{I}_{s V}^{11} & -a_{1} \mathbf{I}_{p V}^{1} \mathbf{I}_{s V}^{12} \\
-a_{2} \mathbf{I}_{p V}^{2} \mathbf{I}_{s V}^{21} & \mathbf{I}^{2}-a_{2} \mathbf{I}_{p V}^{2} \mathbf{I}_{s V}^{22}
\end{array}\right]\left\{\begin{array}{c}
\mathbf{q}^{1} \\
\mathbf{q}^{2}
\end{array}\right\}} \\
& =\left\{\begin{array}{l}
\mathbf{Y}_{V}^{1}+a_{1} \mathbf{I}_{p V}^{1} \mathbf{w}_{s}^{1} \\
\mathbf{Y}_{V}^{2}+a_{2} \mathbf{I}_{p V}^{2} \mathbf{w}_{s}^{2}
\end{array}\right\}, \\
& {\left[\begin{array}{cc}
\mathbf{I}^{1}-b_{1} \mathbf{I}_{p H}^{1} \mathbf{I}_{s H}^{11} & -b_{1} \mathbf{I}_{p H}^{1} \mathbf{I}_{s H}^{12} \\
-b_{2} \mathbf{I}_{p H}^{2} \mathbf{I}_{s H}^{21} & \mathbf{I}^{2}-b_{2} \mathbf{I}_{p H}^{2} \mathbf{I}_{s H}^{22}
\end{array}\right]\left\{\begin{array}{l}
\mathbf{p}^{1} \\
\mathbf{p}^{2}
\end{array}\right\}} \\
& =\left\{\begin{array}{l}
\mathbf{Y}_{H}^{1}+b_{1} \mathbf{I}_{p H}^{1} \mathbf{u}_{s}^{1} \\
\mathbf{Y}_{H}^{2}+b_{2} \mathbf{I}_{p H}^{2} \mathbf{u}_{s}^{2}
\end{array}\right\},
\end{aligned}
$$

where $a_{1}=d_{1} n_{1}^{2} E_{p 1} / 4 L_{1}^{2} ; a_{2}=d_{2} n_{2}^{2} E_{p 2} / 4 L_{2}^{2} ; b_{1}=n_{1}^{4} E_{p 1} I_{p 1} /$ $L_{1}^{4} ; b_{2}=n_{2}^{4} E_{p 2} I_{p 2} / L_{2}^{4} ; E_{p 1}$ and $E_{p 2}$ are the elastic modulus of piles 1 and 2, respectively; $d_{1}$ and $d_{2}$ are the diameters of piles 1 and $2 ; L_{1}$ and $L_{2}$ are the lengths of piles 1 and $2 ; n_{1}$ and $n_{2}$ are the total number of the elements along piles 1 and $2 ; I_{p 1}$ and $I_{p 2}$ are the moments of inertia of piles 1 and $2 ; \mathbf{I}^{1}$ and $\mathbf{I}^{2}$ are unit matrices for piles 1 and $2 ; \mathbf{I}_{p V}^{1}$ and $\mathbf{I}_{p V}^{2}$ are the vertical pile flexibility matrices of piles 1 and $2 ; \mathbf{I}_{p H}^{1}$ and $\mathbf{I}_{p H}^{2}$ are the horizontal pile flexibility matrices of piles 1 and $2 ; \mathbf{q}^{1}$ and $\mathbf{q}^{2}$ are the vertical nodal soil resistance force vectors of piles 1 and $2 ; \mathbf{p}^{1}$ and $\mathbf{p}^{2}$ are the horizontal nodal soil resistance force vectors of piles 1 and $2 ; \mathbf{Y}_{V}^{1}=\left\{\begin{array}{llllll}2 Q_{1} n_{1} / \pi d_{1} L_{1} & 0 & \cdots & 0 & 0\end{array}\right\}$, $\mathbf{Y}_{H}^{1}=\left\{\left(2 L_{1} n_{1} T_{1}+2 n_{1}^{2} M_{1}\right) / L_{1}^{2}-n_{1}^{2} M_{1} / L_{1}^{2} \quad 0 \quad \cdots \quad 0\right\}, \mathbf{Y}_{V}^{2}=$ $\left\{2 Q_{2} n_{2} / \pi d_{2} L_{2} \quad 0 \quad \cdots \quad 0 \quad 0\right\}$, and $\mathbf{Y}_{H}^{2}=\left\{\left(2 L_{2} n_{2} T_{2}+2 n_{2}^{2} M_{2}\right) /\right.$ $\begin{array}{lllll}L_{2}^{2} & -n_{2}^{2} M_{2} / L_{2}^{2} & 0 & \cdots & 0\end{array} ; ; \mathbf{w}_{s}^{1}$ and $\mathbf{w}_{s}^{2}$ are the vectors of the vertical free-field soil deformation induced by tunnelling at the location of piles 1 and $2 ; \mathbf{u}_{s}^{1}$ and $\mathbf{u}_{s}^{2}$ are the vectors of the horizontal free-field soil deformation induced by tunnelling at the location of piles 1 and $2 ; \mathbf{I}_{s V}^{11}$ and $\mathbf{I}_{s V}^{22}$ are the vertical soil flexibility matrices of piles 1 and $2 ; \mathbf{I}_{s H}^{11}$ and $\mathbf{I}_{s H}^{22}$ are the horizontal soil flexibility matrices of piles 1 and 2; and $\mathbf{I}_{s V}^{12}$ and $\mathbf{I}_{s V}^{21}$ are the vertical soil-effect matrices between piles 1 and 2 . 


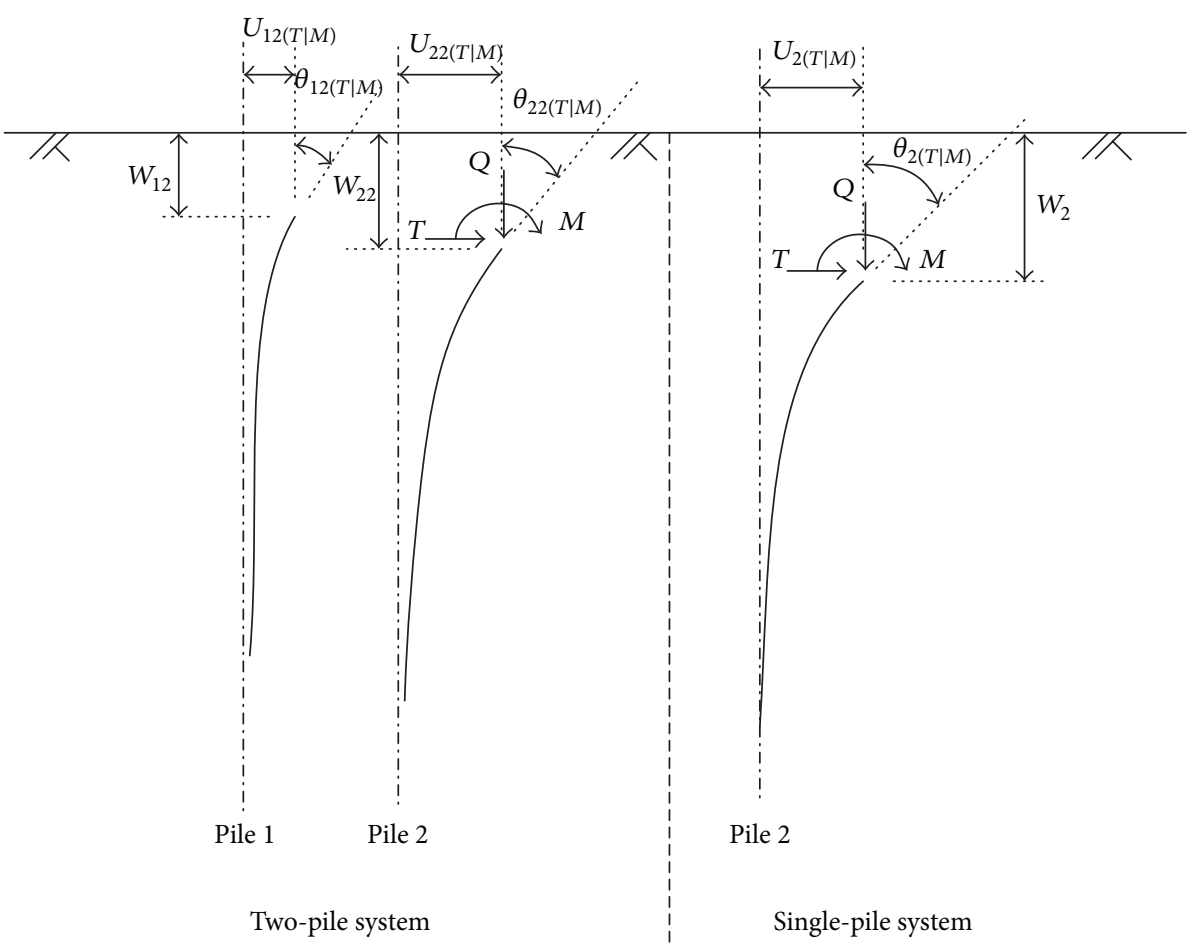

FIgURE 4: Pile-pile interactions.

The element in the $k$ th row and $l$ th column in the matrix $\mathbf{I}_{s V}^{12}$, $I_{s V, k l}^{12}$, represents the vertical soil deformation at the $k$ th node of the soil around pile 1 caused by a unit vertical load acting on the $l$ th node of the soil around pile 2. Elements in matrices $\mathbf{I}_{s V}^{21}, \mathbf{I}_{s H}^{12}$, and $\mathbf{I}_{s H}^{21}$ are alike.

Employing the pile-pile interaction factors defined by the author in a previous paper [21], and as shown in Figure 4, the following pile-pile interactions can be obtained through (6):

$$
\begin{aligned}
& \alpha_{w \mathrm{Q}}^{12}=\frac{w_{12(\mathrm{Q})}}{w_{2(\mathrm{Q})}}, \\
& \alpha_{u T}^{12}=\frac{U_{12(T)}}{U_{2(T)}}, \\
& \alpha_{u M}^{12}=\frac{U_{12(M)}}{U_{2(M)}}, \\
& \alpha_{\theta T}^{12}=\frac{\theta_{12(T)}}{\theta_{2(T)}}, \\
& \alpha_{\theta M}^{12}=\frac{\theta_{12(M)}}{\theta_{2(M)}}, \\
& \omega_{w \mathrm{Q}}^{12}=\frac{w_{2(\mathrm{Q})}-w_{22(\mathrm{Q})}}{w_{2(\mathrm{Q})}}, \\
& \omega_{u T}^{12}=\frac{U_{2(T)}-U_{22(T)}}{U_{2(T)}},
\end{aligned}
$$

$$
\begin{aligned}
& \omega_{u M}^{12}=\frac{U_{2(M)}-U_{22(M)}}{U_{2(M)}}, \\
& \omega_{\theta T}^{12}=\frac{\theta_{2(T)}-\theta_{22(T)}}{\theta_{2(T)}}, \\
& \omega_{\theta M}^{12}=\frac{\theta_{2(M)}-\theta_{22(M)}}{\theta_{2(M)}}, \\
& \rho_{w(p)}^{12}=\frac{w_{1(p)}-w_{11(p)}}{w_{1(p)}} \\
& \rho_{u(p)}^{12}=\frac{U_{1(p)}-U_{11(p)}}{U_{1(p)}}, \\
& \rho_{\theta(p)}^{12}=\frac{\theta_{1(p)}-\theta_{11(p)}}{\theta_{1(p)}},
\end{aligned}
$$

where $U_{12(T)}$ and $U_{12(M)}$ are the horizontal deformations at the head of pile 1 when a unit horizontal load or a unit moment is applied to pile 2 in a two-pile system; $\theta_{12(T)}$ and $\theta_{12(M)}$ are the rotations at the head of pile 1 when a unit horizontal load or a unit moment is applied to pile 2 in a two-pile system; $w_{12}$ is the vertical deformation at the head of pile 1 when a unit vertical load is applied to pile 2 in a twopile system; $U_{22(T)}$ and $U_{22(M)}$ are the horizontal deformations at the head of pile 2 when a unit horizontal load or a unit moment is applied to pile 2 in a two-pile system; $\theta_{22(T)}$ and $\theta_{22(M)}$ are the rotations at the head of pile 2 when a unit 
horizontal load or a unit moment is applied to pile 2 in a twopile system; $w_{22}$ is the vertical deformation at the head of pile 2 when a unit vertical load is applied to pile 2 in a two-pile system; $U_{2(T)}$ and $U_{2(M)}$ are the horizontal deformations at the head of pile 2 when a unit horizontal load or a unit moment is applied to pile 2 in a single-pile system; $\theta_{2(T)}$ and $\theta_{2(M)}$ are the rotations at the head of pile 2 when a unit horizontal load or a unit moment is applied to pile 2 in a single-pile system; $w_{2}$ is the vertical deformation at the head of pile 2 when a unit vertical load is applied to pile 2 in a singlepile system; $w_{1(p)}$ is the vertical deformation of pile 1 when pile 1 is subjected to free-field soil deformations induced by tunnelling without considering the existence of pile $2 ; w_{11(p)}$ is the vertical deformation of the pile head of pile 1 when pile 1 and pile 2 are subjected to free-soil deformations induced by tunnelling. The definitions of the horizontal deformations, $U_{1(p)}, U_{11(p)}$ and rotations, $\theta_{1(p)}, \theta_{11(p)}$, are alike. $w_{2}, U_{2(T)}$, $U_{2(M)}, \theta_{2(T)}, \theta_{2(M)}, w_{1(p)}, U_{1(p)}$, and $\theta_{1(p)}$ can be obtained from (8) and (9), while the other deformations mentioned in this paragraph can be obtained from (6).

3.3. Pile-Soil and Soil-Soil Interactions. The vertical and horizontal equilibrium equations for the pile-soil surface system shown in Figure 5 can be written as follows:

$$
\begin{aligned}
& \left(\mathbf{I}^{1}-a_{1} \mathbf{I}_{p V}^{1} \mathbf{I}_{s V}^{11}\right) \mathbf{q}^{1}=a_{1} \mathbf{I}_{p V}^{1} \mathbf{w}_{s}^{1}+\mathbf{Y}_{V}^{1}+a_{1} \mathbf{I}_{p V}^{1} \mathbf{I}_{V}^{p s} Q_{s}, \\
& \left(\mathbf{I}^{1}-b_{1} \mathbf{I}_{p H}^{1} \mathbf{I}_{s H}^{11}\right) \mathbf{p}^{1}=b_{1} \mathbf{I}_{p L}^{1} \mathbf{u}_{s}^{1}+\mathbf{Y}_{H}^{1}+b_{1} \mathbf{I}_{p H}^{1} \mathbf{I}_{H}^{p s} T_{s},
\end{aligned}
$$

where $\mathbf{I}_{V}^{p s}$ and $\mathbf{I}_{H}^{p s}$ are the vertical and horizontal soil-effect vectors between the pile and soil surface elements. The element in the $k$ th row in the vector $\mathbf{I}_{V}^{p s}, I_{V, k}^{p s}$, represents the vertical soil movement at the $k$ th node of the soil around the pile caused by a unit vertical load acting on the surface of the soil element. The elements in the matrix $\mathbf{I}_{H}^{p s}$ are alike.

Thus, the following pile-soil surface interaction factors can be obtained through (8) and (9):

$$
\begin{gathered}
\kappa_{w Q}^{j i}=\frac{w_{s p(Q)}}{w_{s(Q)}}, \\
\kappa_{u T}^{j i}=\frac{U_{s p(T)}}{U_{s(T)}}, \\
\kappa_{u M}^{j i}=\frac{U_{s p(M)}}{U_{s(T)}}, \\
\beta_{w Q}^{i j}=\frac{w_{p s(Q)}}{w_{2(Q)}}, \\
\beta_{u T}^{i j}=\frac{U_{p s(T)}}{U_{2(T)}}, \\
\beta_{\theta T}^{i j}=\frac{\theta_{p s(T)}}{\theta_{2(T)}},
\end{gathered}
$$

$$
\begin{aligned}
\zeta_{w q}^{j i} & =\frac{w_{s(Q)}-w_{s(Q) p}}{w_{s(Q)}}, \\
\zeta_{u p}^{j i} & =\frac{U_{s(T)}-U_{s(T) p}}{U_{s(T)}}, \\
\gamma_{w(p)}^{j i} & =\frac{w_{s(p)}-w_{s(p) p}}{w_{s(p)}}, \\
\gamma_{u(p)}^{j i} & =\frac{U_{s(p)}-U_{s(p) p}}{U_{s(p)}},
\end{aligned}
$$

where $w_{s p(Q)}$ is the vertical deformation of the soil surface element when a unit vertical load is acting on the head of the pile; $U_{s p(T)}$ is the horizontal deformation of the soil surface element when a unit horizontal load is acting on the head of the pile; $U_{s p(M)}$ is the horizontal deformation of the soil surface element when a unit moment is acting on the head of the pile; $w_{p s(Q)}$ is the vertical deformation of the pile head when a unit vertical load is acting on the soil surface element; $U_{p s(T)}$ is the horizontal deformation of the pile head when a unit horizontal load is acting on the soil surface element; $\theta_{p s T}$ is the rotation of the pile head when a unit horizontal load is acting on the soil surface element; $w_{s(\mathrm{Q})}$ is the vertical deformation of the soil surface element when a unit vertical load is acting on the soil surface element without considering the existence of the pile; $w_{s(\mathrm{Q}) p}$ is the vertical deformation of the soil surface element when a unit vertical load is acting on the soil surface element that considers the existence of the pile; $U_{s(T)}$ is the horizontal deformation of the soil surface element when a unit horizontal load is acting on the soil surface element without considering the existence of the pile; $U_{s(T) p}$ is the horizontal deformation of the soil surface element when a unit horizontal load is acting on the soil surface element which considers the existence of the pile; $w_{s(p)}$ is the vertical free-field soil deformation at the soil surface element without considering the pile, which can be obtained through $(2) ; w_{s(p) p}$ is the vertical deformation of the soil surface element induced by tunnelling that considers the existence of the pile; $U_{s(p)}$ is the horizontal free-field soil deformation at the soil surface element without considering the pile, which can be obtained through (1); and $U_{s(p) p}$ is the horizontal deformation of the soil surface element induced by tunnelling that considers the existence of the pile. The superscripts " $i$ " and " $j$ " in (10)-(17) denote the $i$ th pile and the $j$ th soil surface element, respectively.

Using the solutions of the asymmetric problem in multilayered systems presented by Mu et al. [13], we can now obtain the soil surface-soil surface interaction (shown in Figure 5) factors, $\xi_{w q}^{j g}$ and $\xi_{u T}^{j g}$.

3.4. Pile-Raft Foundation. Assuming that no separation occurs between the raft and the soil, the compatibility condition between the raft and the foundation, which includes the soil elements and piles shown in Figure 1, can be summarized as follows: (1) the deformations of pile heads or of the soil surface elements are the same as the deformations of the corresponding elements of the raft and (2) the summations of the internal forces between the elements of the raft and the 


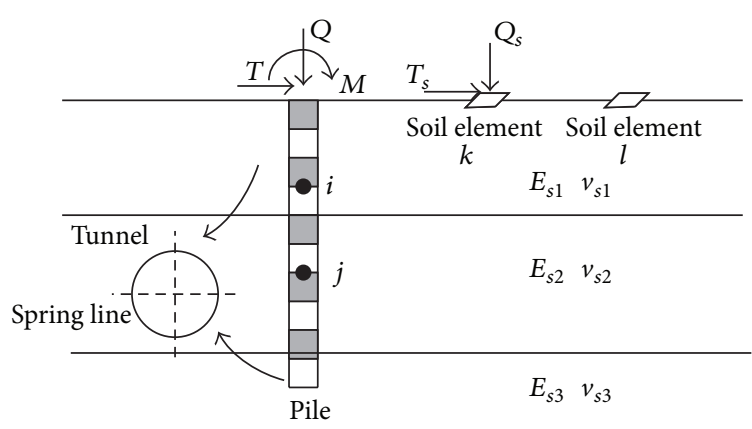

FIgURE 5: Pile-soil-tunnel system.

elements of the foundation, including piles and soil surface elements, are equal to the external loads acting on the raft. These can be written in equation form as follows:

$$
\begin{aligned}
u_{1}+u_{1 p}= & \cdots=u_{i}+u_{i p}=\cdots \\
= & u_{m+n}+u_{(m+n) p}, \\
\theta_{1}+\theta_{1 p}= & \cdots=\theta_{i}+\theta_{i p}=\cdots \\
= & \theta_{m+n}+\theta_{(m+n) p}, \\
w_{1}+w_{1 p}= & w_{2}+w_{2 p}+x_{2}\left(\theta_{2}+\theta_{2 p}\right)=\cdots \\
= & w_{i}+w_{i p}+x_{i}\left(\theta_{i}+\theta_{i p}\right)=\cdots \\
= & w_{m+n}+w_{(m+n) p} \\
& +x_{m+n}\left[\theta_{m+n}+\theta_{(m+n) p}\right]
\end{aligned}
$$

$$
\begin{aligned}
\sum_{i=1}^{m+n} T_{i} & =T, \\
\sum_{i=1}^{m} M_{i}+\sum_{i=1}^{m+n} Q_{i} x_{i} & =M, \\
\sum_{i=1}^{m+n} Q_{i} & =Q,
\end{aligned}
$$

where $u_{i}$ is the horizontal deformation of the $i$ th element induced by the internal force between the $i$ th element of the raft and the corresponding pile or soil surface element; $u_{i p}$ is the horizontal deformation of the $i$ th element induced by tunnelling; $\theta_{i}$ is the rotation of the $i$ th element induced by the internal force between the $i$ th element of the raft and the corresponding pile or soil surface element; $\theta_{i p}$ is the rotation of the $i$ th element induced by tunnelling; $w_{i}$ is the vertical deformation of the $i$ th element induced by the internal force between the $i$ th element of the raft and the corresponding pile or soil surface element; $w_{i p}$ is the vertical deformation of the $i$ th element induced by tunnelling; $x_{i}$ is the $x$-coordinate of the $i$ th element; and $T_{i}, M_{i}$, and $Q_{i}$ are the internal forces between the $i$ th element of the raft and the corresponding piles or soil surfaces.

Thus, the equilibrium equation for the pile-raft foundation-tunnel system can be written in matrix form as follows:

$$
\left\{\begin{array}{c}
\mathbf{E}_{(m \times 1)} \\
\mathbf{F}_{(n \times 1)} \\
\mathbf{K}_{(3 \times 1)}
\end{array}\right\}=\left[\begin{array}{ccc}
\mathbf{A}_{(m \times m)} & \mathbf{B}_{(m \times n)} & -\mathbf{J}_{(1 \times m)}^{T} \\
\mathbf{C}_{(n \times m)} & \mathbf{D}_{(n \times n)} & \mathbf{R}_{(m \times 1)} \\
\mathbf{J}_{(1 \times m)} & \mathbf{I}_{(1 \times n)} & \mathbf{0}
\end{array}\right]\left\{\begin{array}{c}
\mathbf{P}_{(m \times 1)} \\
\mathbf{N}_{(n \times 1)} \\
\mathbf{E E}_{(3 \times 1)}
\end{array}\right\},
$$

where

$$
\begin{aligned}
\mathbf{E}=\left[\begin{array}{l}
u_{1} \\
\theta_{1} \\
w_{1}
\end{array}\right], \\
\mathbf{P}_{i}=\left[\begin{array}{l}
T_{i} \\
M_{i} \\
Q_{i}
\end{array}\right], \\
\mathbf{N}_{j}=\left[\begin{array}{l}
T_{j} \\
Q_{j}
\end{array}\right], \\
\mathbf{E}_{i}=\left[\begin{array}{c}
u_{1 p}\left(1-\sum_{i=2}^{m} \rho_{u(p)}^{1 i}\right)-u_{i p}\left(1-\sum_{j=1, j \neq i}^{m} \rho_{u(p)}^{i j}\right) \\
w_{1 p}\left(1-\sum_{i=2}^{m} \rho_{w(p)}^{1 i}\right)-w_{i p}\left(\begin{array}{c}
m \\
\theta_{1 p}\left(1-\sum_{j=1, j \neq i}^{m} \rho_{\theta(p)}^{1 i}\right.
\end{array}\right)-\theta_{i p}\left(1-\sum_{j=1, j \neq i}^{m} \rho_{\theta(p)}^{i j}\right)+\theta_{1 p}\left(1-\sum_{i=2}^{m} \rho_{\theta(p)}^{1 i}\right)\left(x_{i}-x_{1}\right)
\end{array}\right],
\end{aligned}
$$




$$
\begin{aligned}
& \mathbf{F}_{j}=\left[\begin{array}{c}
u_{1 p}\left(1-\sum_{i=2}^{m} \rho_{u(p)}^{1 i}\right)-u_{j p}\left(1-\sum_{i=1}^{m} \gamma_{u(p)}^{j i}\right) \\
w_{1 p}\left(1-\sum_{i=2}^{m} \rho_{w(p)}^{1 i}\right)-w_{j p}\left(1-\sum_{i=1}^{m} \gamma_{w(p)}^{j i}\right)+\theta_{1 p}\left(1-\sum_{i=2}^{m} \rho_{\theta(p)}^{1 i}\right)\left(x_{j}-x_{1}\right)
\end{array}\right], \\
& \mathbf{K}=\left[\begin{array}{c}
T \\
M \\
Q
\end{array}\right], \\
& \mathbf{I}_{j}=-\mathbf{R}_{j}^{T}=\left[\begin{array}{cc}
1 & 0 \\
0 & x_{j}-x_{1} \\
0 & 1
\end{array}\right] \text {, } \\
& \mathbf{J}_{i}=\left[\begin{array}{ccc}
1 & 0 & 0 \\
0 & 1 & x_{j}-x_{1} \\
0 & 0 & 1
\end{array}\right], \\
& \mathbf{A}_{i j}=\left\{\begin{array}{lll}
{\left[\begin{array}{ccc}
\alpha_{u T}^{i j} U_{2(T)} & \alpha_{u M}^{i j} U_{2(M)} & 0 \\
\alpha_{\theta T}^{i j} \theta_{2(T)} & \alpha_{\theta M}^{i j} \theta_{2(M)} & 0 \\
0 & 0 & \alpha_{w Q}^{i j} w_{2(Q)}
\end{array}\right]} \\
{\left[\begin{array}{ccc}
\alpha_{u T}^{i j} U_{2(T)} & \alpha_{u M}^{i j} U_{2(M)} & 0 \\
\alpha_{\theta T}^{i j} \theta_{2(T)} & \alpha_{\theta M}^{i j} \theta_{2(M)} & 0 \\
0 & 0 & \alpha_{w Q}^{i j} w_{2(Q)}
\end{array}\right]-\sum_{k=1, k \neq i}^{m}\left[\begin{array}{ccc}
\omega_{u T}^{i k} U_{2(T)} & \omega_{u M}^{i k} U_{2(M)} & 0 \\
\omega_{\theta T}^{i k} \theta_{2(T)} & \omega_{\theta M}^{i k} \theta_{2(M)} & 0 \\
0 & 0 & \omega_{w Q}^{i k} w_{2(Q)}
\end{array}\right] \quad i=j,}
\end{array}\right. \\
& \mathbf{B}_{i j}=\left[\begin{array}{cc}
\beta_{u T}^{i j} U_{2(T)} & 0 \\
\beta_{\theta T}^{i j} \theta_{2(T)} & 0 \\
0 & \beta_{w Q}^{i j} w_{2(Q)}
\end{array}\right] \text {, } \\
& \mathbf{C}_{i j}=\left[\begin{array}{ccc}
\kappa_{u T}^{i j} U_{s T} & \kappa_{u M}^{i j} U_{s T} & 0 \\
0 & 0 & \kappa_{w Q^{j j}} w_{s Q}
\end{array}\right] \text {, } \\
& \mathbf{D}_{i j}= \begin{cases}{\left[\begin{array}{cc}
\xi_{u T}^{i j} U_{s T} & 0 \\
0 & \xi_{w Q}^{i j} w_{s Q}
\end{array}\right]} & i \neq j \\
{\left[\begin{array}{cc}
\xi_{u T}^{i j} U_{s T} & 0 \\
0 & \xi_{w Q}^{i j} w_{s Q}
\end{array}\right]-\sum_{k=1}^{m}\left[\begin{array}{cc}
\zeta_{u T}^{i k} U_{s T} & 0 \\
0 & \zeta_{w Q}^{i k} w_{s Q}
\end{array}\right]} & i=j,\end{cases}
\end{aligned}
$$

where $\mathbf{A}_{i j}, \mathbf{B}_{i j}, \mathbf{C}_{i j}$, and $\mathbf{D}_{i j}$ are the pile-pile, pile-soil surface, soil surface-pile, and soil surface-soil surface interaction matrices, respectively.

\section{Verification of Our Method}

An APPR program was developed that was based on our proposed method. APPR is verified through comparisons with results from centrifuge test, other analytical methods, and finite-element method.
4.1. A Single Pile Subjected to Active and Passive Loads. Reference [19] carried out a series of centrifuge tests to investigate the responses of loaded single piles induced by tunnelling. The length of the pile was $27 \mathrm{~m}$, the diameter of the pile was $1.06 \mathrm{~m}$, and the modulus and Poisson's ratio of the pile were $20 \mathrm{GPa}$ and 0.3 , respectively. The diameter of the tunnel was $6 \mathrm{~m}$. Using the method proposed herein, we calculated a case where the depth of the tunnel spring line was $21 \mathrm{~m}$, the ground-loss ratio was $1 \%$, and the working load was $0 \mathrm{kN}, 1600 \mathrm{kN}$, and $3200 \mathrm{kN}$, as shown in Figure 6. According 


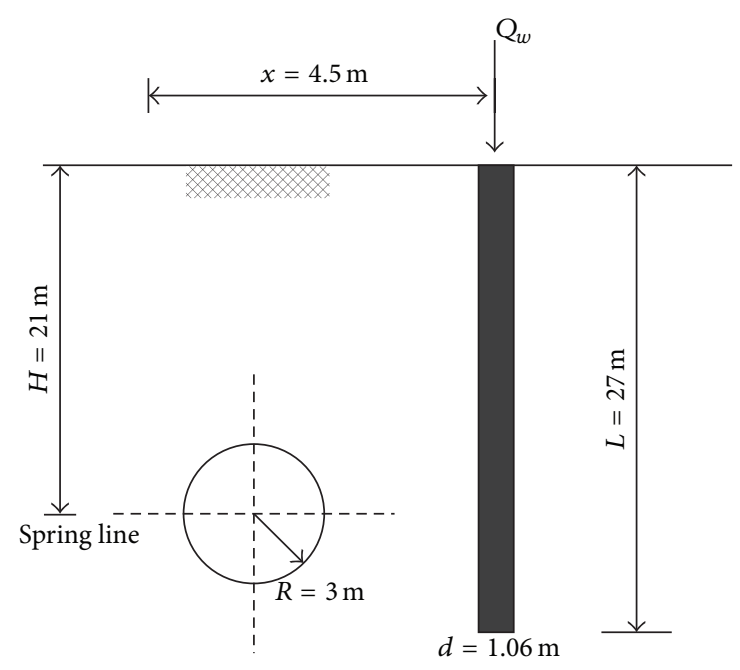

FIGURE 6: Sketch of the centrifuge test.

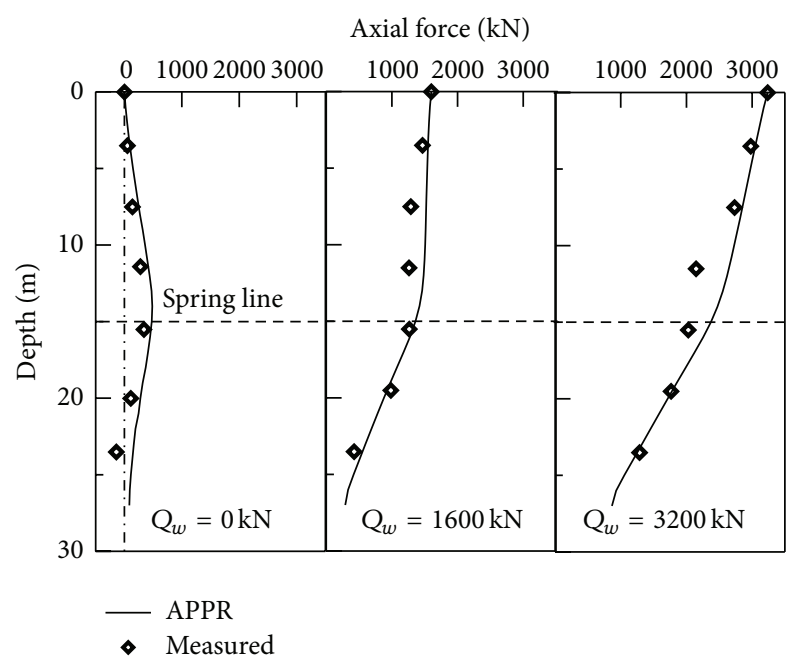

(a) Axial force

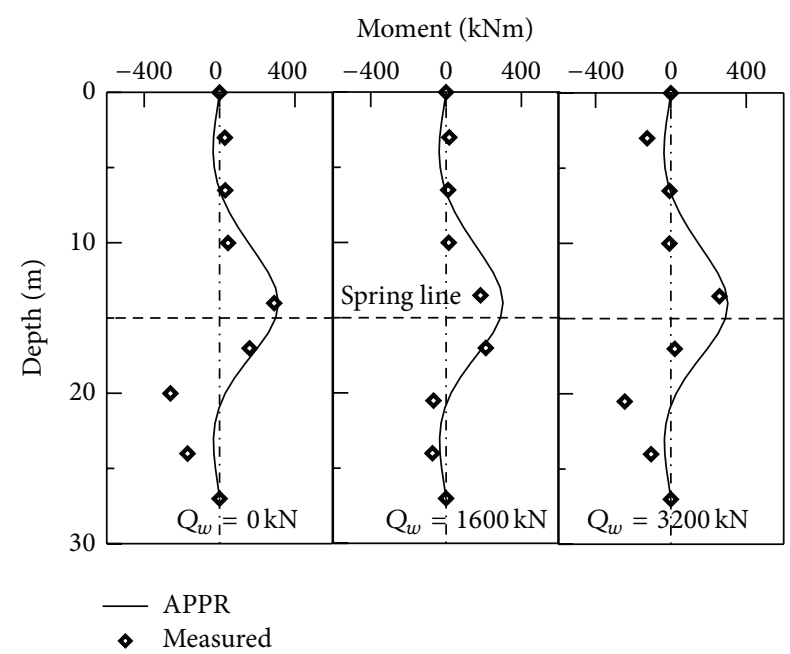

(b) Moment

FIGURE 7: Comparison of the centrifuge test results.

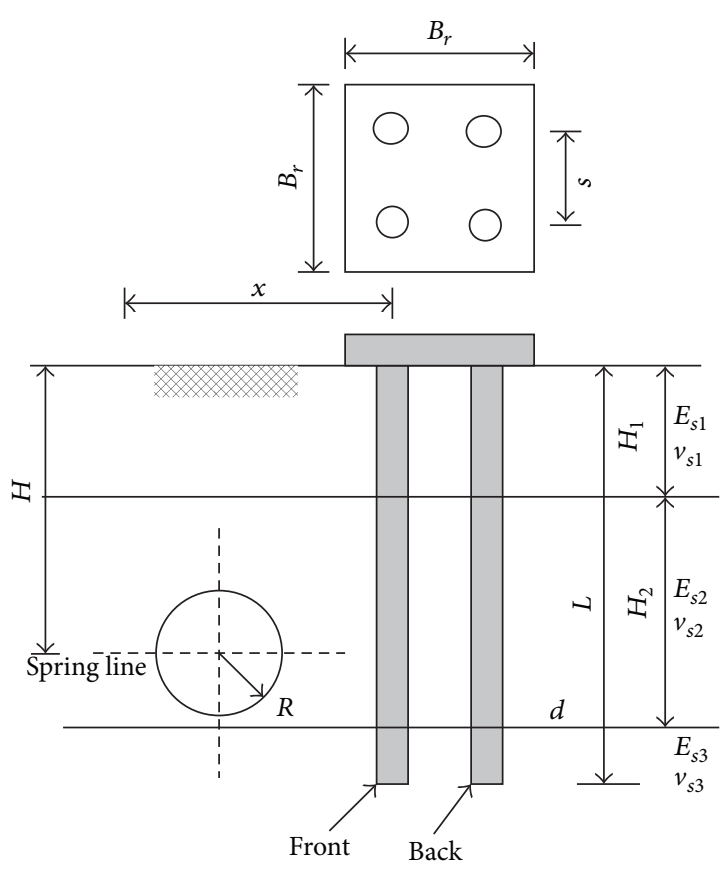

FIGURE 8: A pile-raft foundation subject to both active and passive loads.

to [19], when the working load was smaller than $6700 \mathrm{kN}$, the responses of the pile were nearly linear elastic, and when the settlement of the pile head reached $0.106 \mathrm{~m}$, the load on the pile head was $6700 \mathrm{kN}$. Thus, using the optimization tool in MATLAB and (8), the soil modulus was found to be $4 \mathrm{MPa}$, and Poisson's ratio of the soil was found to be 0.4 . The results are shown in Figure 7. It can be seen that the calculated results agree well with the results from the centrifuge test, both with and without a working load acting on the pile.

4.2. A Pile-Raft Foundation Subjected to an Active Load. Since no results have been published regarding the multidirectional responses of a pile-raft foundation subjected to both active and passive loads, APPR was first used to calculate the responses of the pile-raft foundation under pure active loads and pure passive loads separately to verified the ability of APPR to evaluate this problem. Then, APPR was used to calculate the responses of a multidirectionally loaded pile-raft foundation subjected to tunnelling and compared with results from FEM. As shown in Figure 8, the pile-raft foundation was only subjected to an active load combined with a vertical load, a horizontal load, and a moment. In this case, $E_{s 1}: E_{s 2}: E_{s 3}=$ $1: 2: 4, v_{s 1}=v_{s 2}=v_{s 3}=0.5, s=1.5 \mathrm{~m}, d=0.4 \mathrm{~m}, L=10 \mathrm{~m}$, $H_{1}=3 \mathrm{~m}, H_{2}=4 \mathrm{~m}$, and $B_{r}=3 \mathrm{~m}$. Two further cases were then calculated: (1) where the loads were applied separately and (2) where the loads were applied simultaneously. The results were compared with the results from $[21,29]$ and FEM. It can be seen from Figure 9, without considering the passive load, that the results from APPR are the same as the results from the method proposed by [21] when the loads were applied simultaneously. Moreover, the results from APRR also agree well with the results from [29] and FEM. 


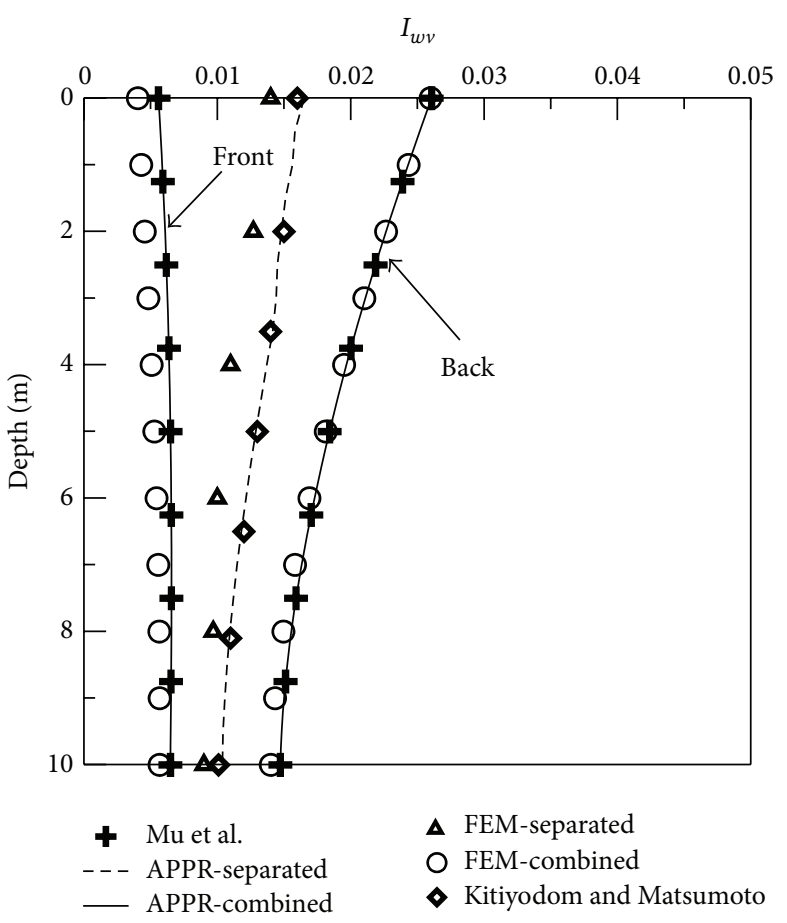

(a) Vertical deformation

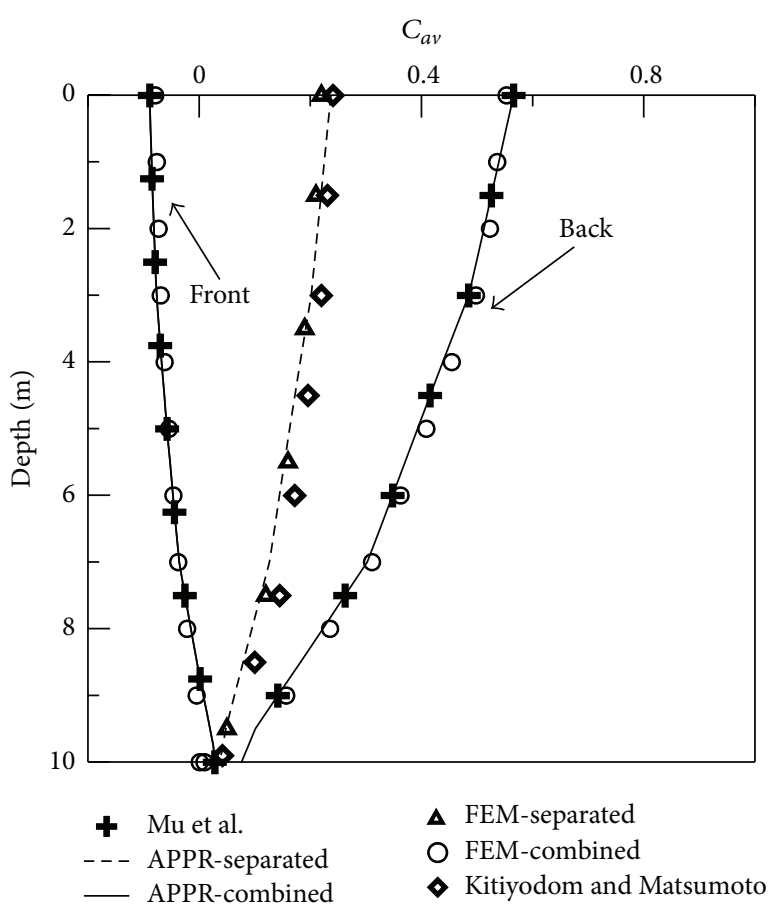

(c) Axial force

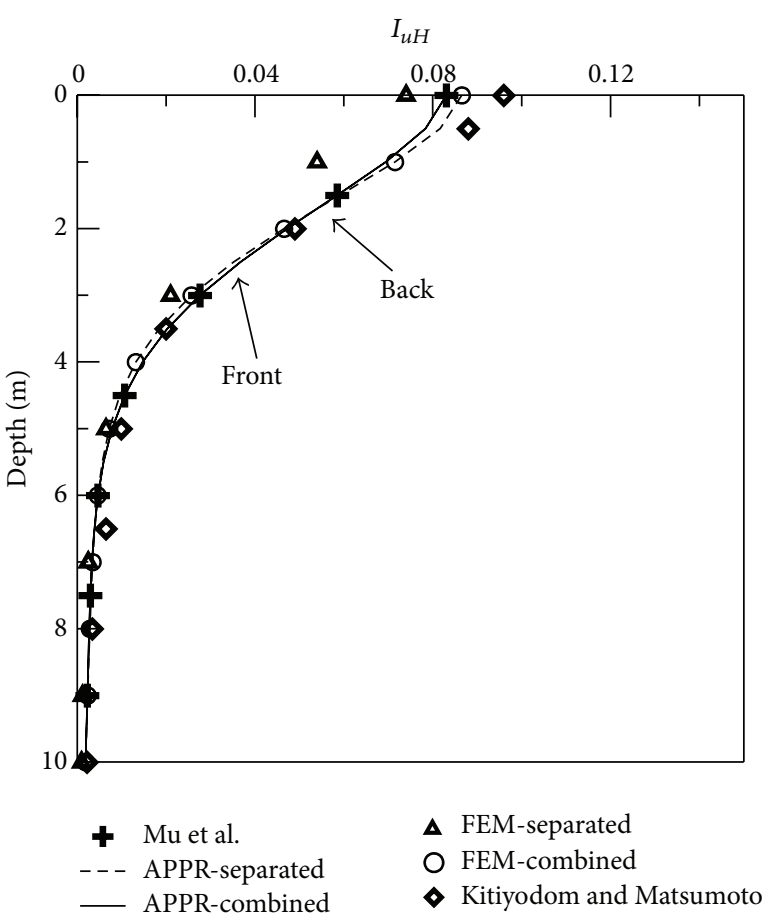

(b) Horizontal deformation

$$
C_{b H}
$$

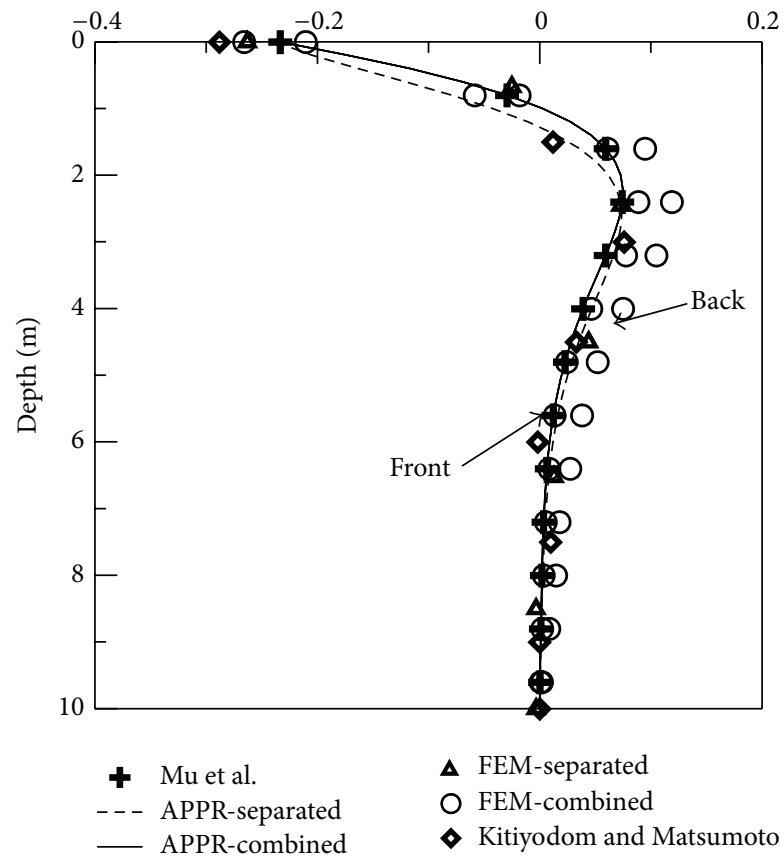

(d) Moment

Figure 9: The responses of a pile-raft foundation under an active load.

4.3. A Pile-Raft Foundation Subjected to a Passive Load. The raft-soil interaction was not considered in our analysis when the pile-raft foundation was only subjected to passive loads because the soil will always separate from the raft for a pileraft foundation subjected to pure passive load induced by tunnelling without considering the self-weights of the raft and the superstructure. In this case, $E_{s 1}: E_{s 2}: E_{s 3}=1: 2: 2$, $v_{s 1}=v_{s 2}=v_{s 3}=0.5, s=2.4 \mathrm{~m}, d=0.8 \mathrm{~m}, L=25 \mathrm{~m}, H=$ $20 \mathrm{~m}, H_{1}=10 \mathrm{~m}, x=4.5 \mathrm{~m}, R=3 \mathrm{~m}, B_{r}=4.8 \mathrm{~m}$, and the ground-loss ratio was set at $1 \%$. The responses are shown in 


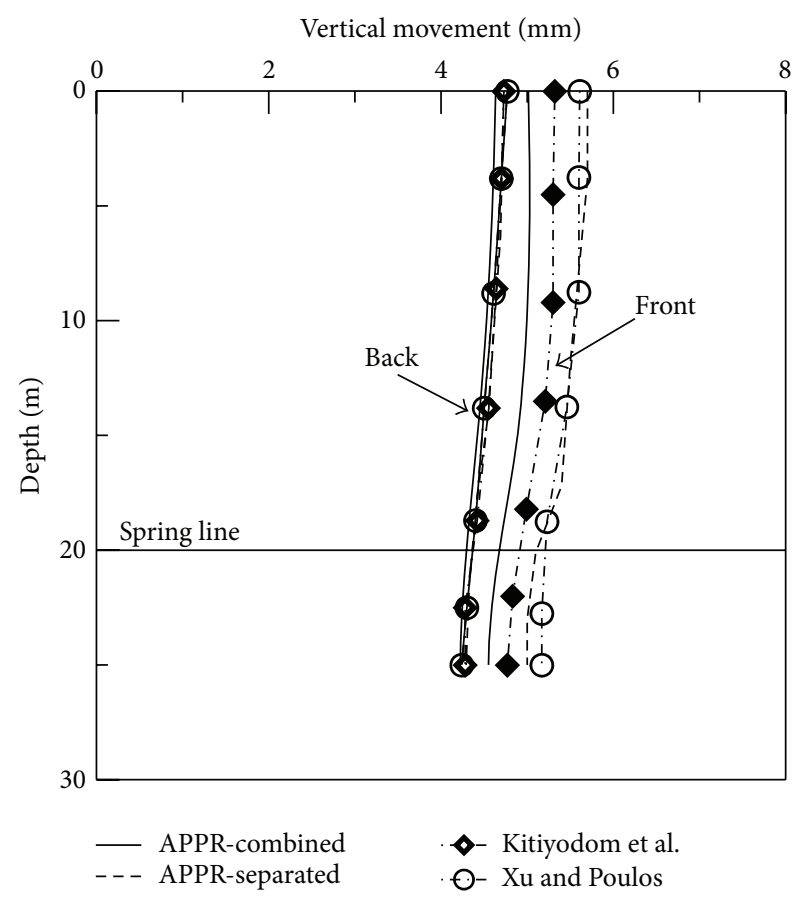

(a) Vertical deformation

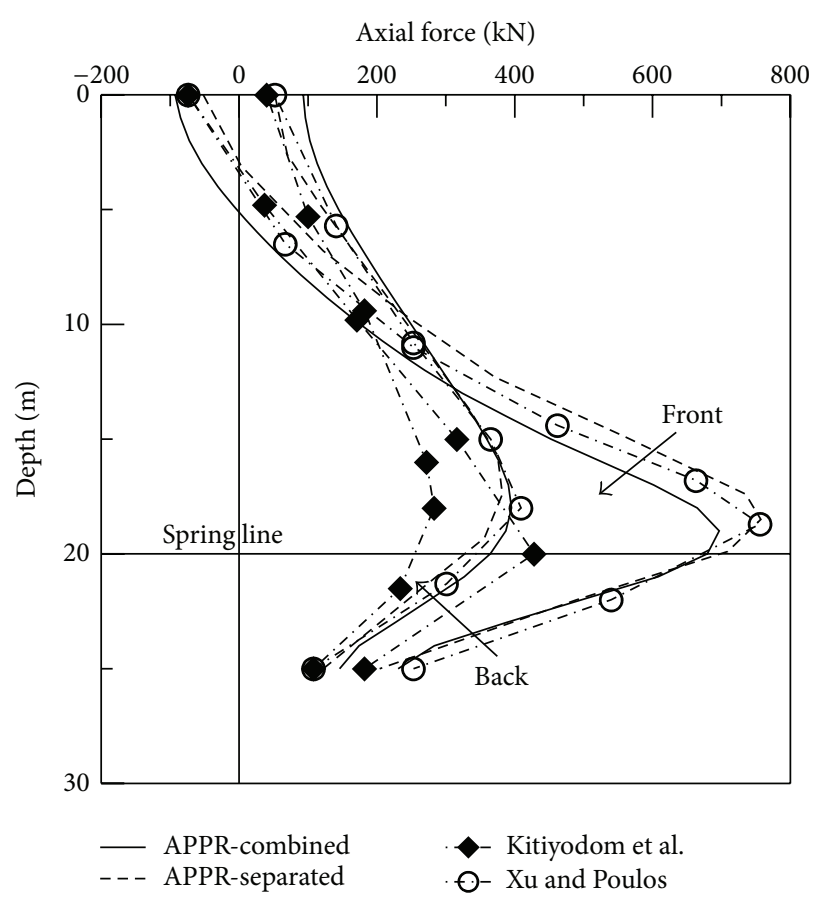

(c) Axial force

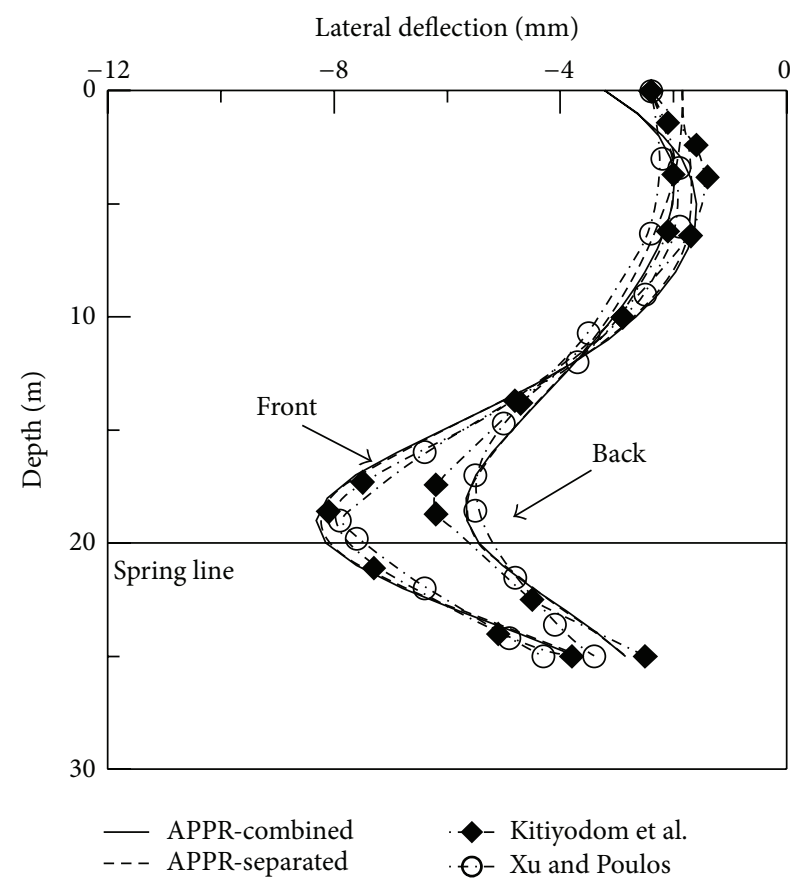

(b) Horizontal deformation

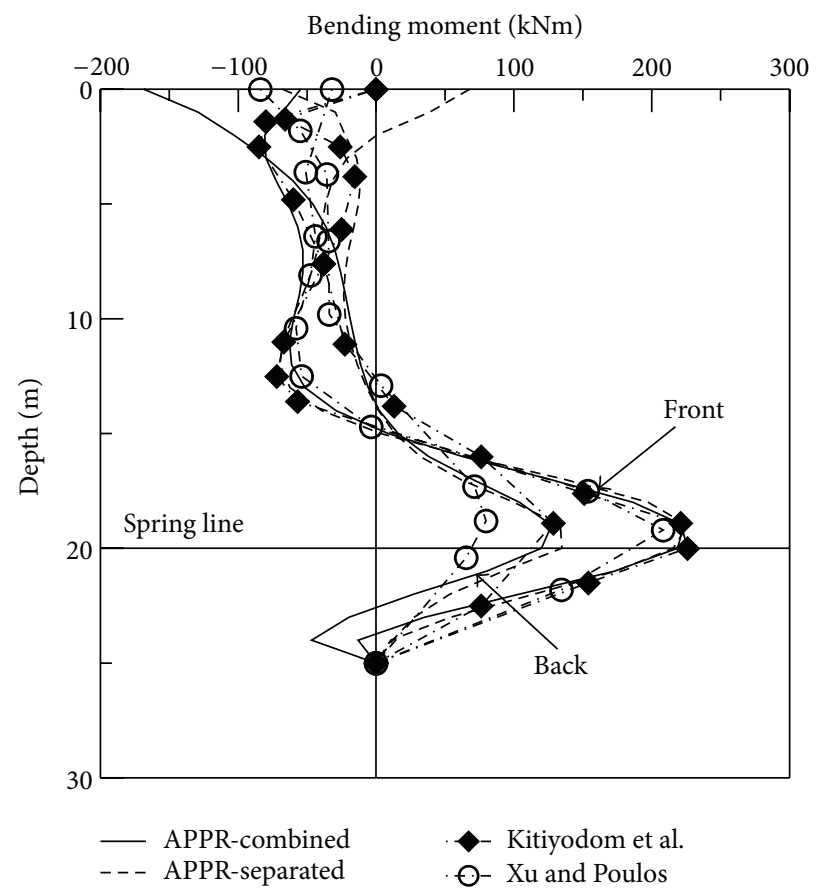

(d) Moment

FIGURE 10: The responses of a pile-raft foundation under a passive load.

Figure 10. The results from the APPR analysis agree well with the results from $[8,11]$, except for those around the head of the piles. This may be due to the different treatments of raft-soil interaction. The internal force between the raft and the soil is a distributed force which is exactly reflected in the proposed method herein. Meanwhile the internal force is assumed to be a point force in [11]. Additionally, it can be shown that the deformation at the pile head will be overestimated, and the internal forces at the pile head will be underestimated, for a pile-raft foundation subjected to soil movement induced by tunnelling if the internal force between the raft and the soil is replaced with a point force. 


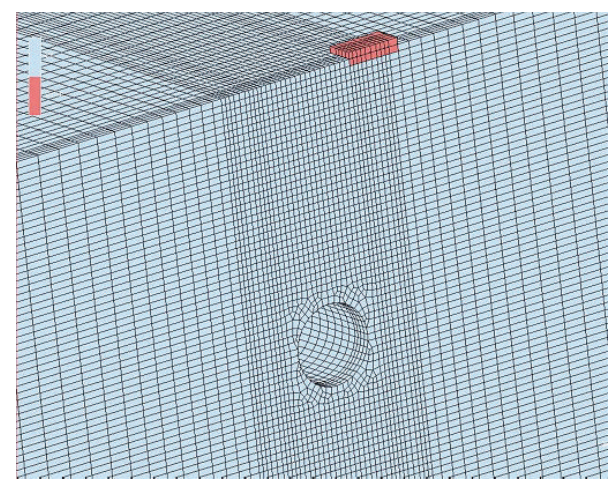

FIGURE 11: Mesh of a pile-raft foundation-tunnel system.

4.4. A Pile-Raft Foundation Subjected to Both Active and Passive Loads in Layered Soil. A finite-element analysis and an APPR analysis were carried out to estimate the responses of the pile-raft foundation that was subjected to both active and passive loads, as shown in Figure 8. In the FEM analysis, the active load on the pile-raft foundation was force controlled, and the passive load was deformation controlled, which also controls the ground-loss ratio. The mesh of the FEM result is shown in Figure 11. Here the parameters of the soil, pileraft foundation, and tunnel are the same as those mentioned in Section 4.3. A comparison of the results from the FEM and APPR analyses is shown in Figure 12. It is seen that it is suitable to use APPR to calculate the coupled responses of a pile-raft foundation subjected to both multidirectional active and passive loads in layered soil.

\section{Parametric Study}

After careful verification, APPR was used to calculate the responses of a pile-raft under both active and passive loads to study the influence of tunnelling on a pile-raft when different working loads act upon the raft. As shown in Figure 8, $E_{s 1}=$ $12 \mathrm{MPa}, E_{s 2}=E_{s 3}=24 \mathrm{MPa}, H_{1}=10 \mathrm{~m}, v_{s 1}=v_{s 2}=v_{s 3}=$ $0.5, L=25 \mathrm{~m}, d=0.8 \mathrm{~m}, s=2.4 \mathrm{~m}, B_{r}=4.8 \mathrm{~m}, H=20 \mathrm{~m}$, $R=3 \mathrm{~m}$, and $\varepsilon_{0}=1 \%$. The raft was subjected to a vertical load, a horizontal load, and a moment separately. Here, the vertical load was set at $0 \mathrm{kN}, 2500 \mathrm{kN}, 5000 \mathrm{kN}$, and $7500 \mathrm{kN}$, the horizontal load was set at $-7500 \mathrm{kN},-5000 \mathrm{kN},-2500 \mathrm{kN}$, $2500 \mathrm{kN}, 5000 \mathrm{kN}$, and $7500 \mathrm{kN}$, and the moment was set at $-7500 \mathrm{kN} \cdot \mathrm{m},-5000 \mathrm{kN} \cdot \mathrm{m},-2500 \mathrm{kN} \cdot \mathrm{m}, 2500 \mathrm{kN} \cdot \mathrm{m}$, $5000 \mathrm{kN} \cdot \mathrm{m}$, and $7500 \mathrm{kN} \cdot \mathrm{m}$. The results are shown in Figures 13, 14, and 15. It can be seen from Figure 13 that, without considering the $p-\Delta$ effect of a single pile, the horizontal deformation and moment along the piles were controlled by tunnelling when there was only a vertical load acting on the raft. However, the vertical deformations of the piles increased with an increase of the vertical load. When the active vertical load was small, the maximum axial force along the pile was controlled by tunnelling, while the active vertical load was large when the maximum axial force was controlled by the active vertical load. Tunnelling had little effect on the axial force, which can be obviously obtained from the responses of the back pile when the vertical load is $7500 \mathrm{kN}$. This means that tunnelling imparted no additional axial force on the piles when the active vertical load reached the ultimate value, which is consistent with the conclusion of [20]. No work has yet been published that studies the responses of a pile-raft foundation induced by tunnelling when the raft is under a horizontal load and moment. It can be obtained from Figures 14 and 15 that the horizontal deformations of the piles, which were below a depth of $15 \mathrm{~m}$, were controlled by the tunnelling when the raft was subjected to a moment, while the horizontal deformations of the piles were controlled by both the horizontal load and tunnelling when the raft was subjected to a horizontal load. The moments of the piles, which were below a depth of $15 \mathrm{~m}$, were controlled by the tunnelling for both the cases in which the raft was subjected to a moment and a horizontal load. When the values of the moment and the horizontal load were the same, the axial force induced by tunnelling was more obvious for the pile-raft foundation that was subjected to a horizontal load than when subjected to a moment. Moreover, when the active loads on the pile-raft foundation increased, the influence of tunnelling became less significant, which agrees with the conclusion obtained from the calculation when the raft was subjected to only a vertical load. It can also be concluded that the influence of tunnelling on pile responses is more obvious when the pileraft foundation was subjected to a moment than when it was subjected to a horizontal load.

In order to evaluate the influence of tunnelling on pileraft foundations under different loads, the following influence factors were defined:

$$
\begin{aligned}
K_{u} & =\frac{u_{t p}}{u_{t}}, \\
K_{R} & =\frac{\theta_{t p}}{\theta_{t}}, \\
K_{\mathrm{AF}} & =\frac{\mathrm{AF}_{\max p}}{\mathrm{AF}_{\max }}, \\
K_{M} & =\frac{M_{\max p}}{M_{\max }},
\end{aligned}
$$

where $u_{t p}$ is the horizontal deformation of the raft induced by tunnelling; $u_{t}$ is the horizontal deformation of the raft induced by both tunnelling and an active load; $\theta_{t p}$ is the rotation of the raft induced by tunnelling; $\theta_{t}$ is the rotation of the raft induced by both tunnelling and an active load; $\mathrm{AF}_{\max p}$ is the maximum axial force along the piles induced by tunnelling; $\mathrm{AF}_{\max }$ is the maximum axial force along the piles induced by both tunnelling and an active load; $M_{\max p}$ is the maximum moment along the piles induced by tunnelling; $M_{\max }$ is the maximum moment along the piles induced by both tunnelling and an active load. The larger the influence factor is, the more necessary it is to consider the influence of tunnelling.

As shown in Figure 16, the rotation and horizontal deformation of the raft were only induced by tunnelling when there was only vertical load acting on the raft, while the axial force was significantly influenced by a vertical load. For a pileraft foundation that was subjected to a horizontal load or 


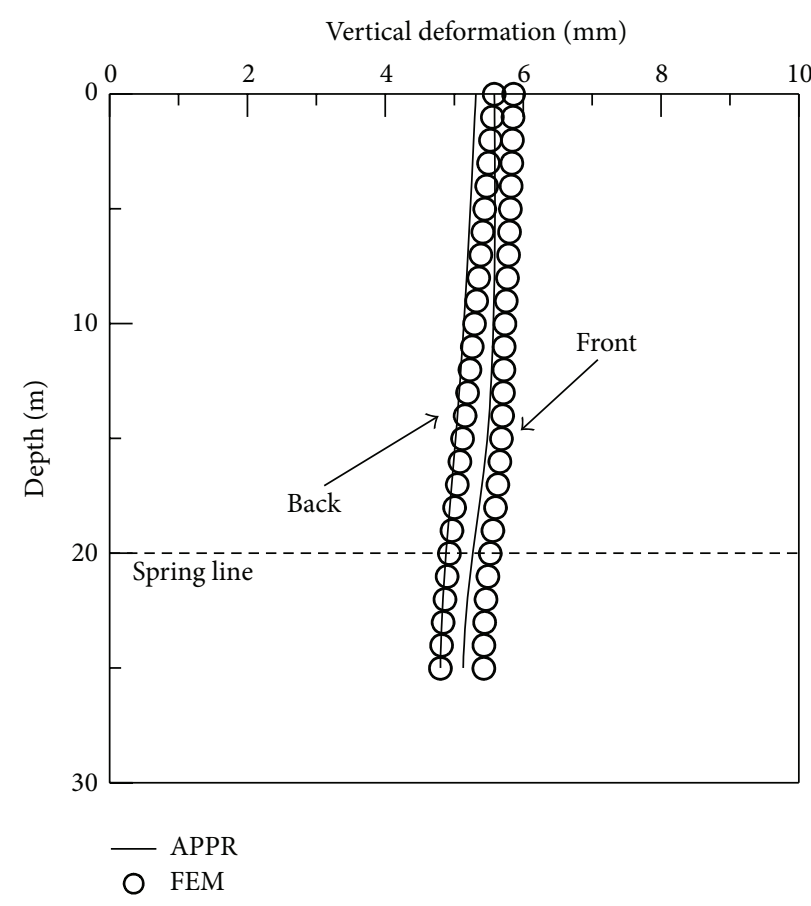

(a) Vertical deformation

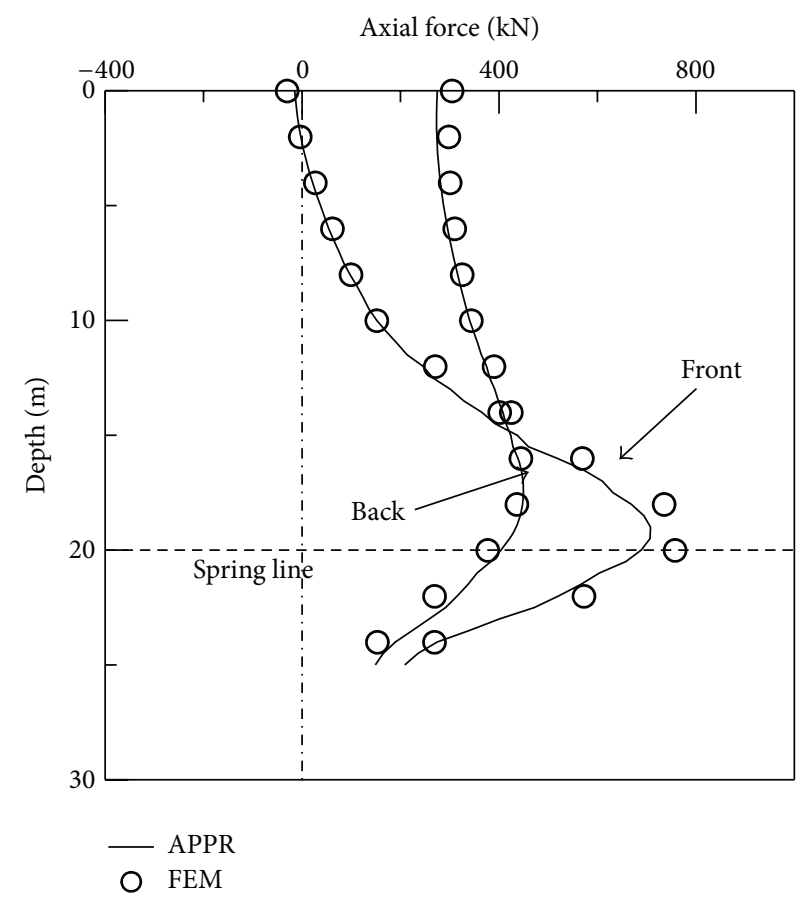

(c) Axial force

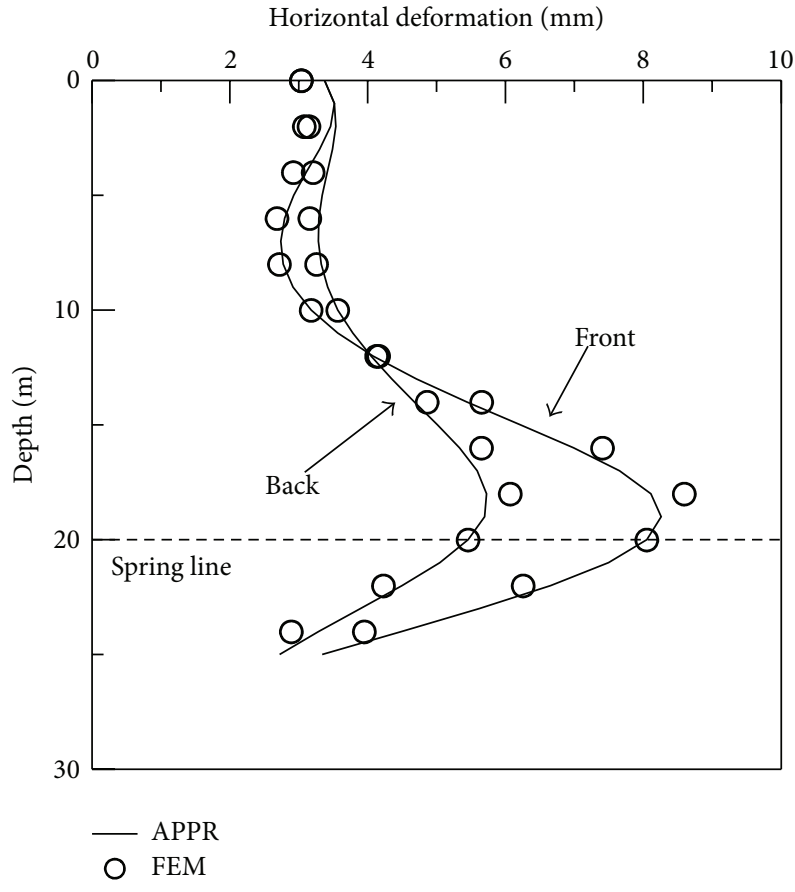

(b) Horizontal deformation

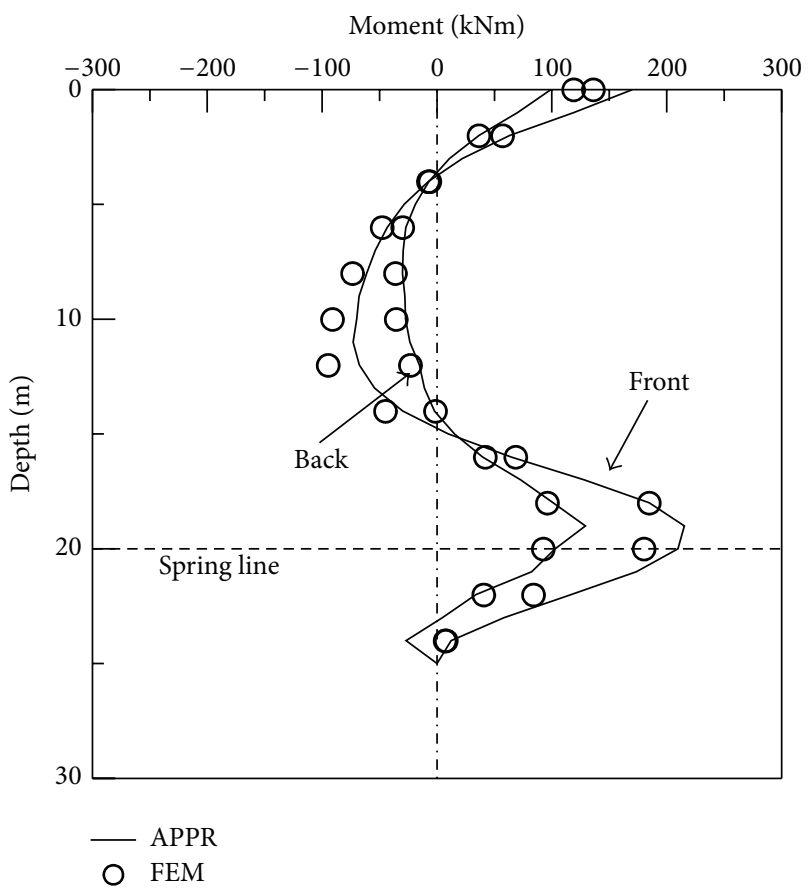

(d) Moment

FIGURE 12: The responses of a pile-raft foundation subjected to active and passive loads.

a moment, the influence factors decreased when the absolute value of the horizontal load or moment increased. When the load was close to 0 , the influence factor was large. The same conclusion can be obtained from Figures 17 and 18. In the cases studied herein, the influence factors decreased with an increase of the absolute value of the loads, when the absolute values of the loads were larger than 5000 .

\section{Conclusions}

Based on asymmetric solutions for layered-elastic half spacing, a modified two-stage method was proposed in this paper to evaluate the coupled responses of multidirectionally loaded pile-raft foundation subjected to tunnelling. Through comparisons with published results from centrifuge tests, 


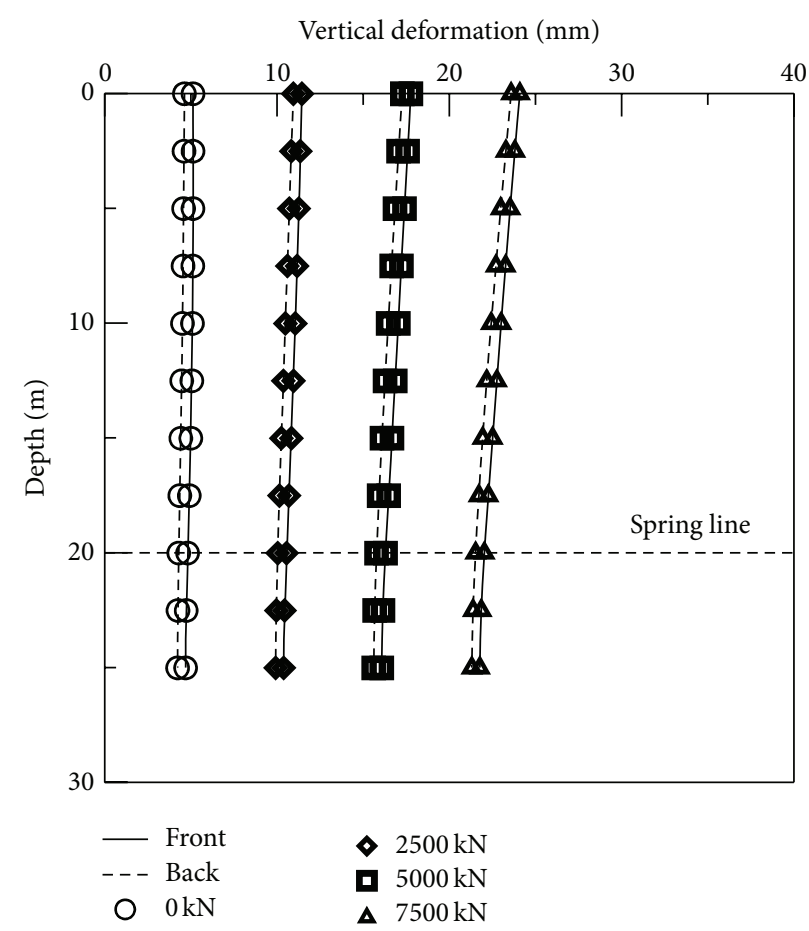

(a) Vertical deformation

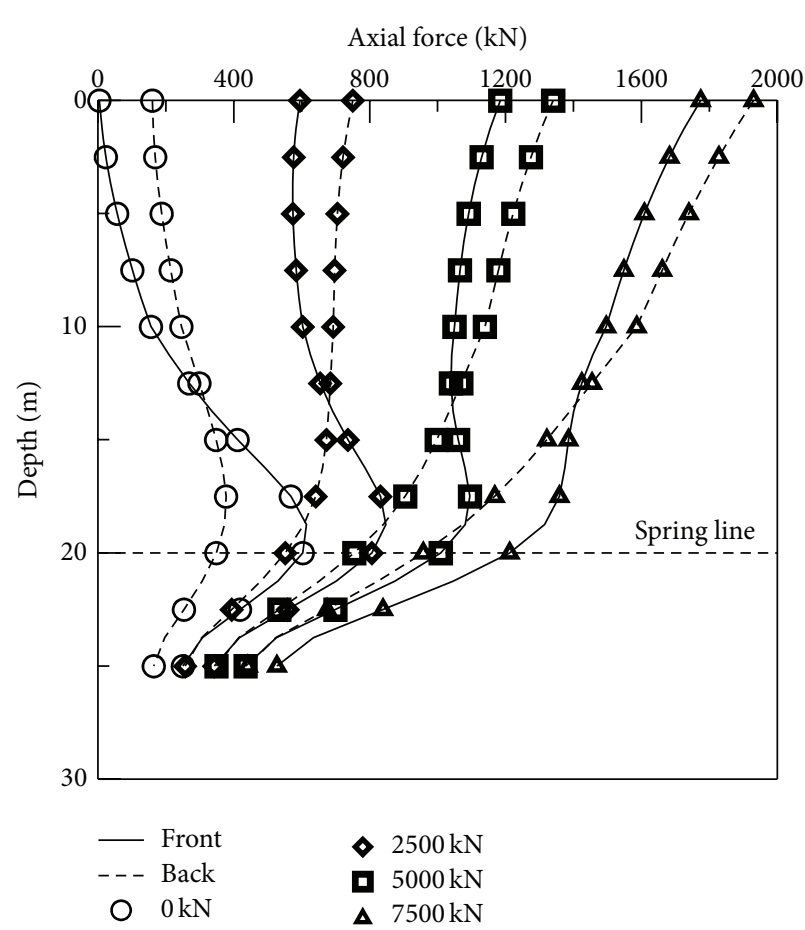

(c) Axial force

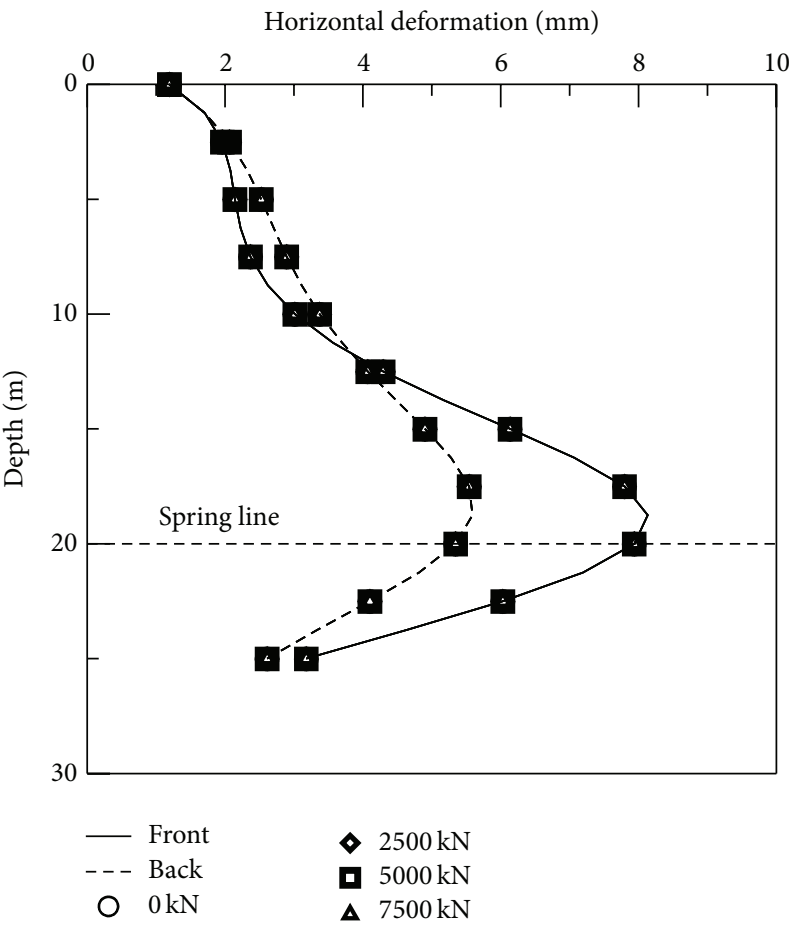

(b) Horizontal deformation

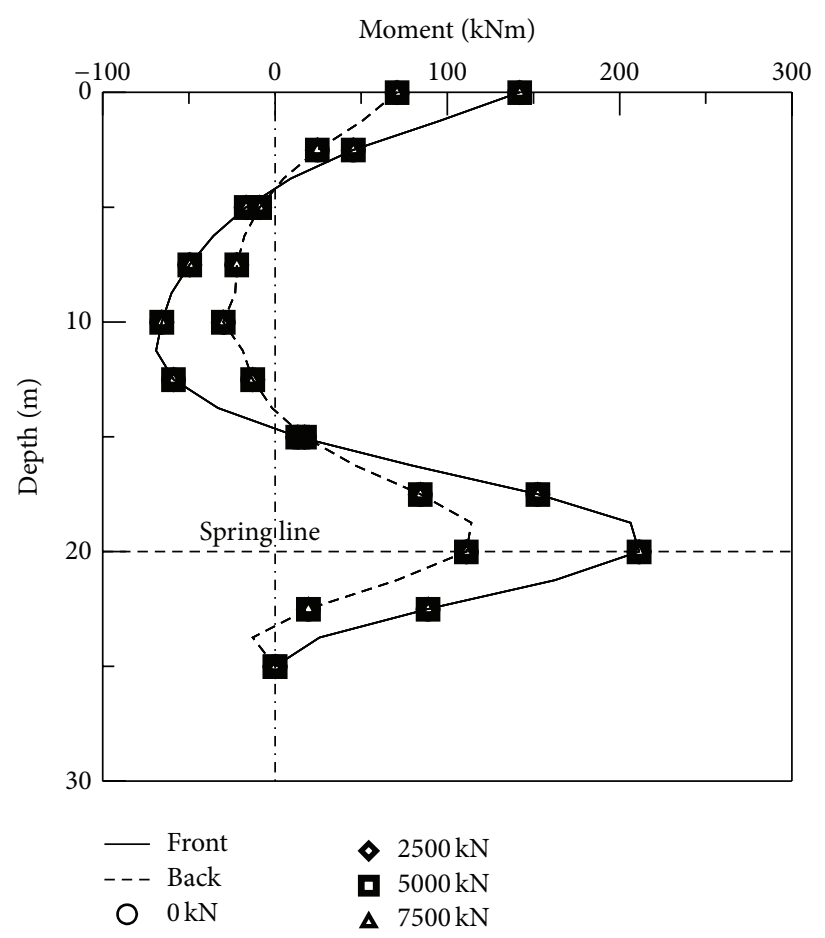

(d) Moment

FIGURE 13: The responses of a pile-raft foundation under a vertical load when subjected to tunnelling. 


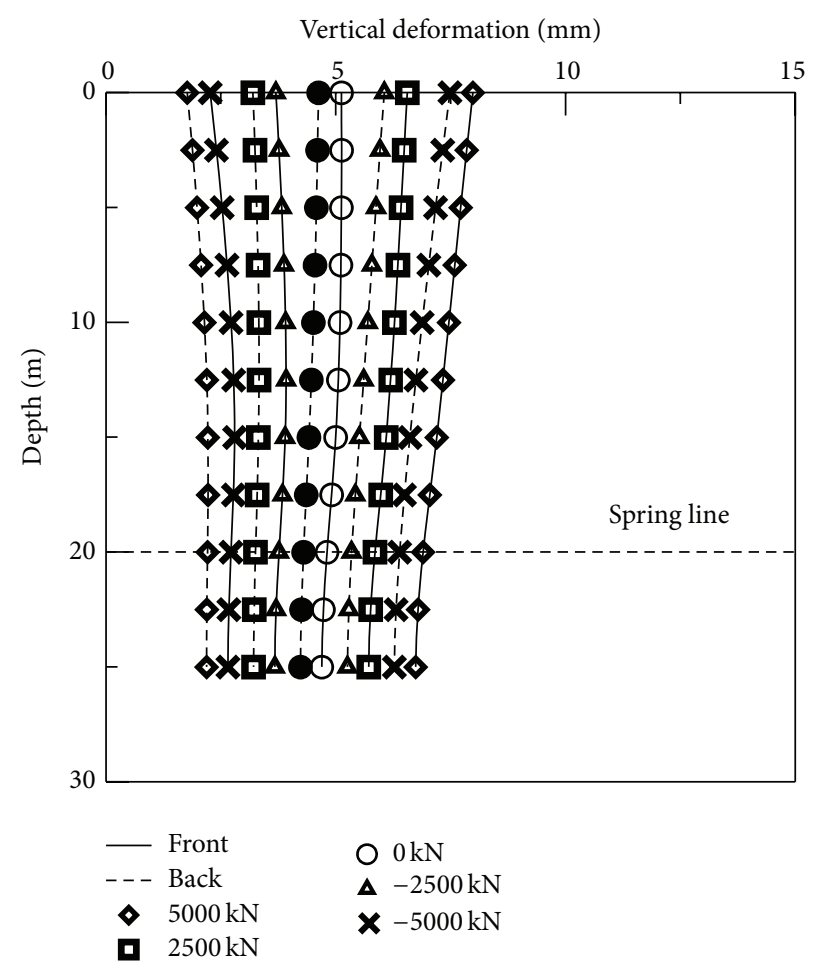

(a) Vertical deformation

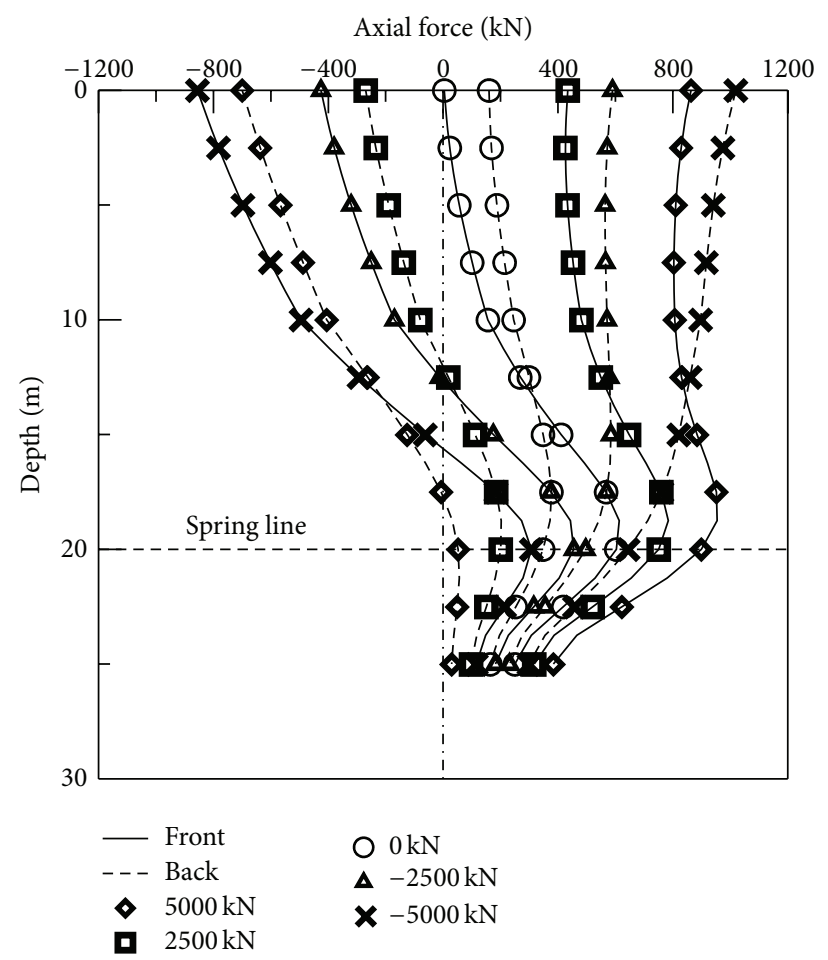

(c) Axial force

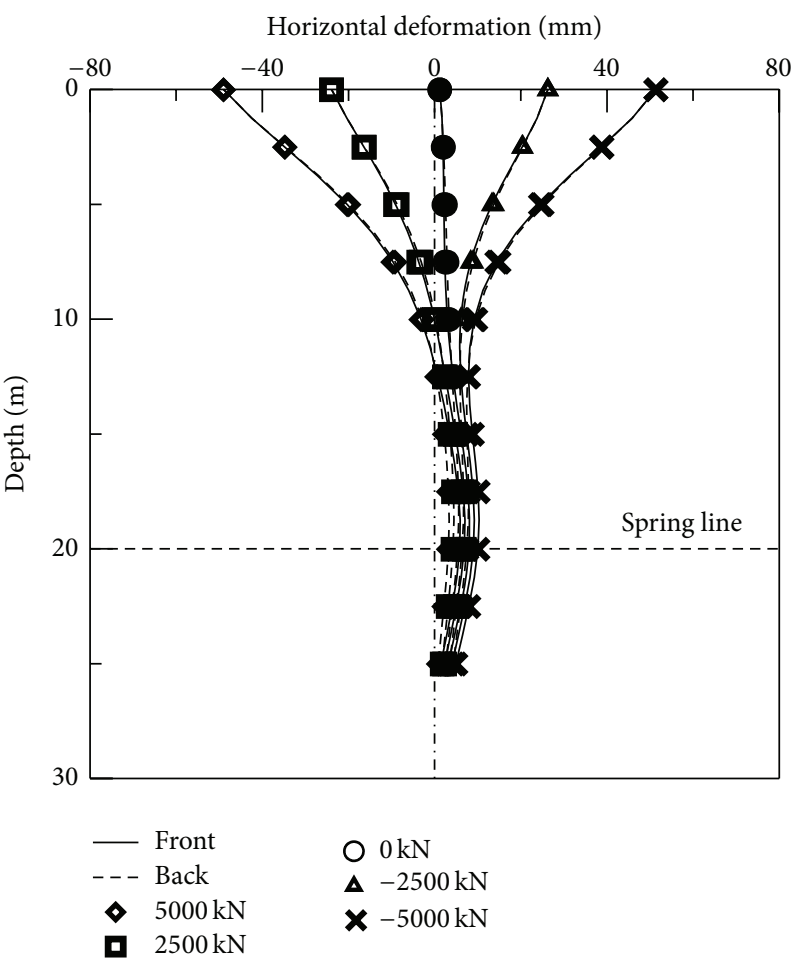

(b) Horizontal deformation

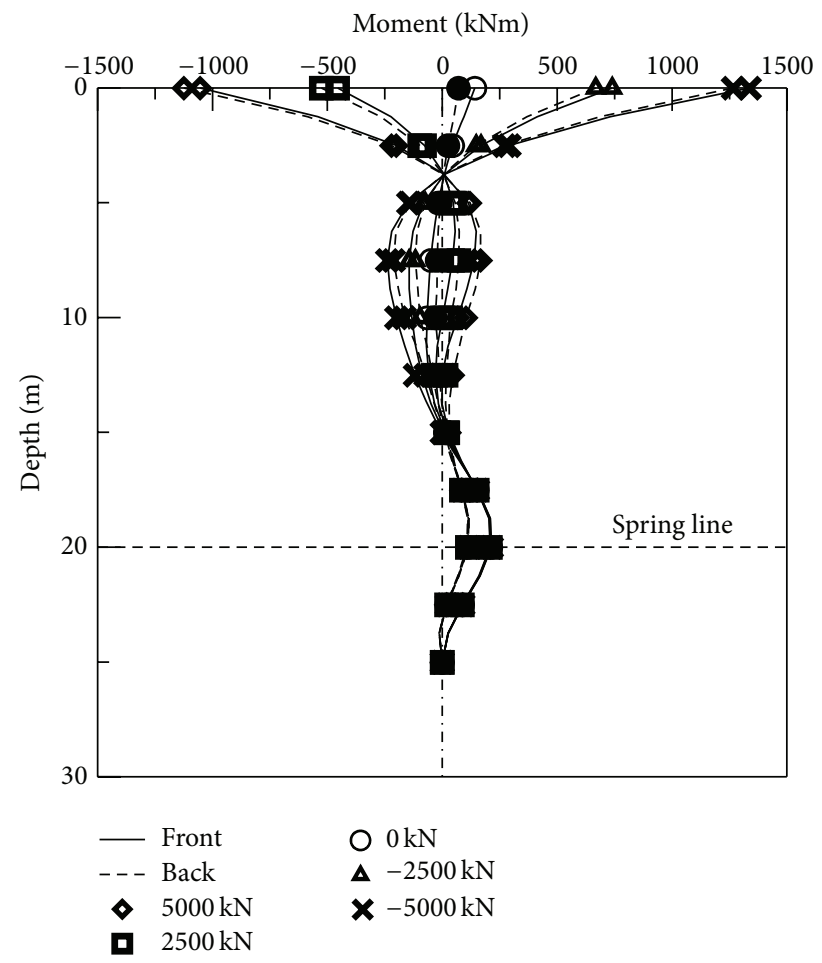

(d) Moment

FIgURE 14: The responses of a pile-raft foundation under a horizontal load when subjected to tunnelling. 
Vertical deformation (mm)

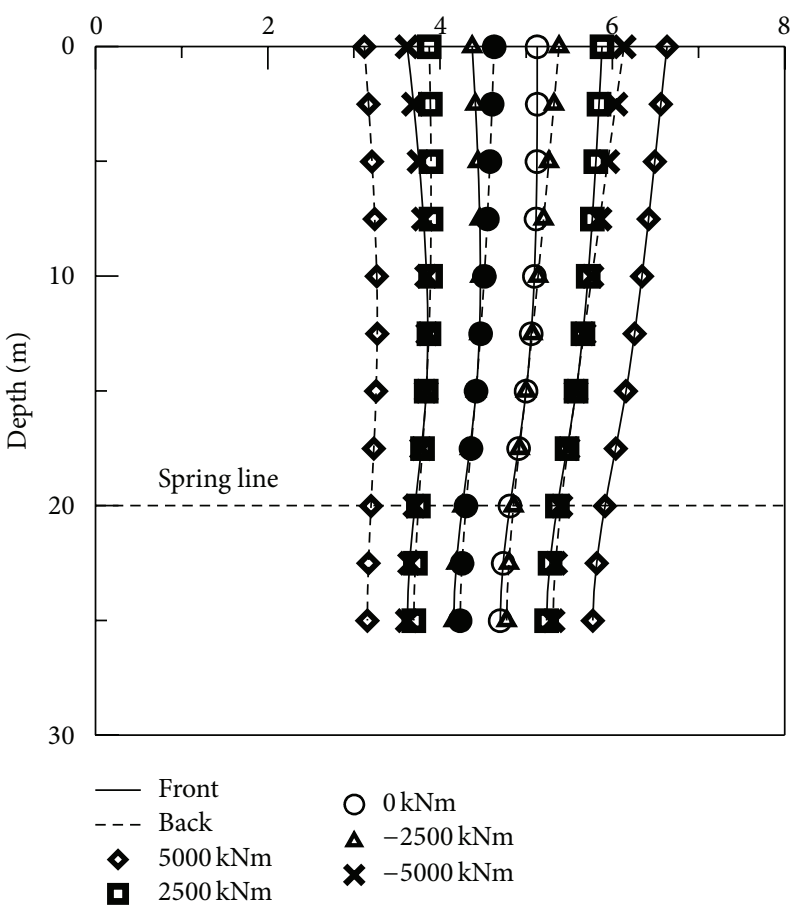

(a) Vertical deformation

Axial force $(\mathrm{kN})$

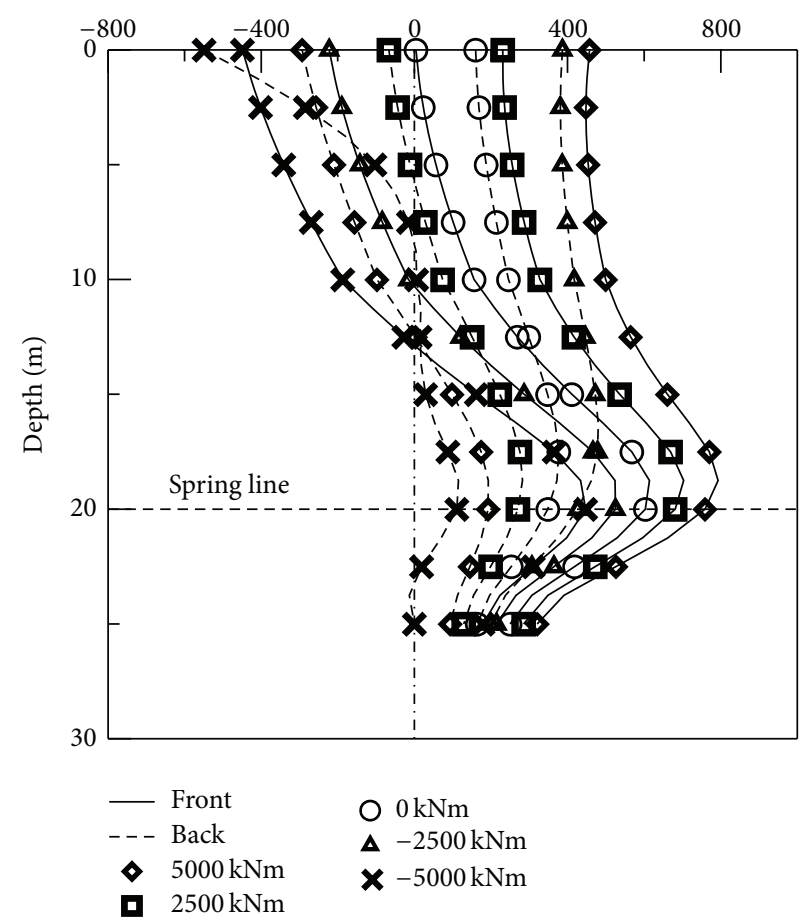

(c) Axial force
Horizontal deformation (mm)

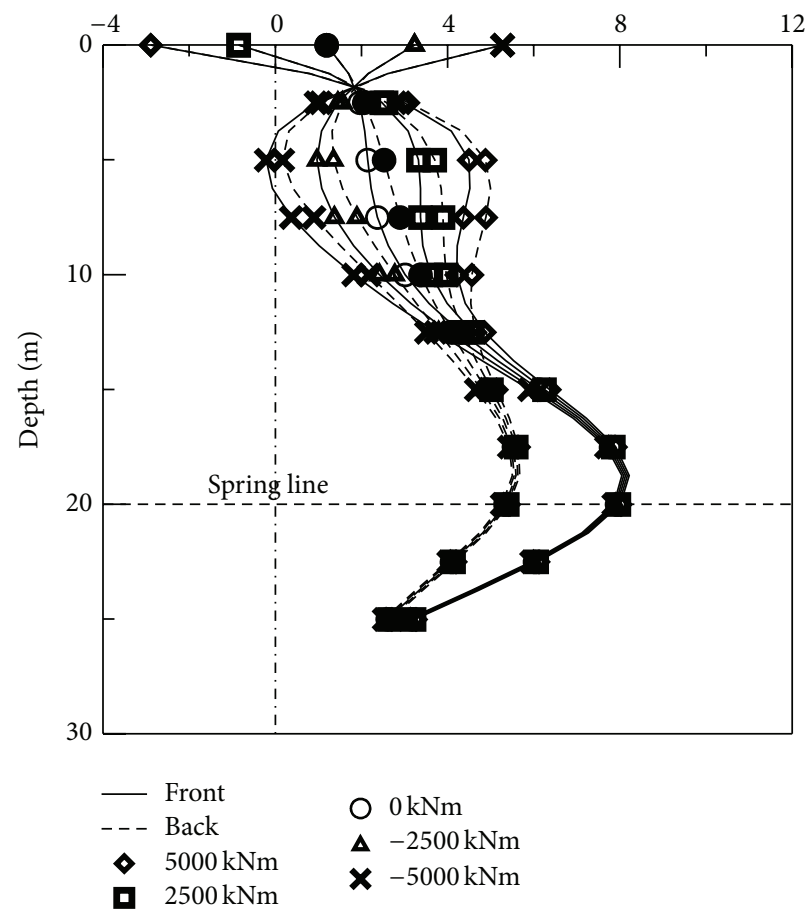

(b) Horizontal deformation

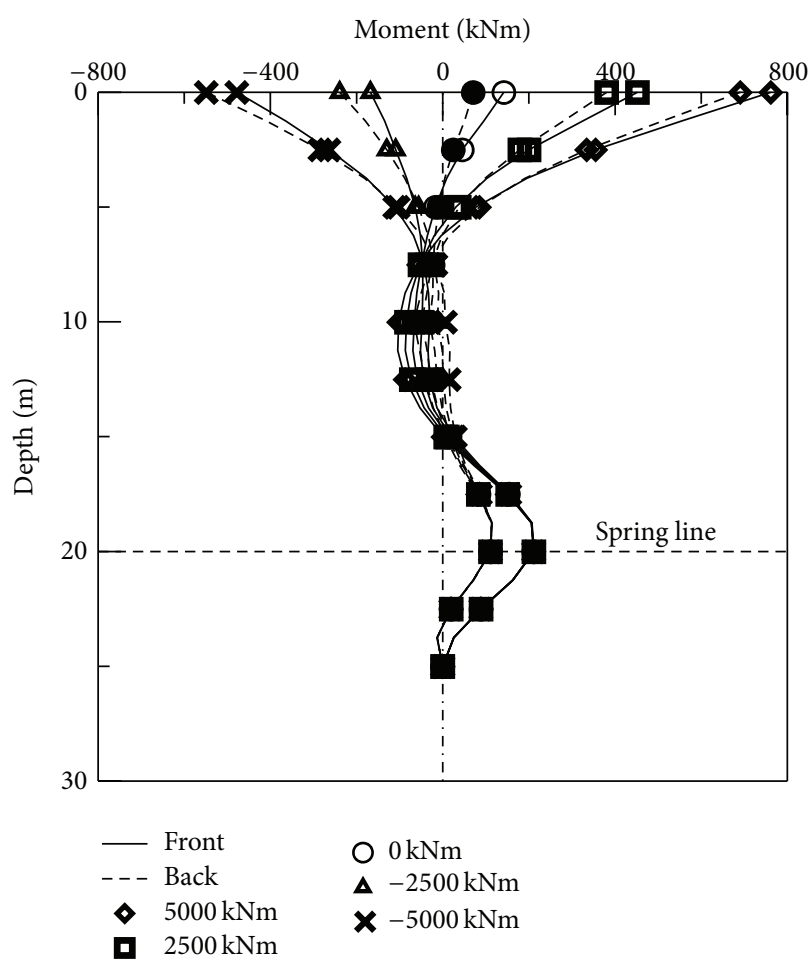

(d) Moment

FIGURE 15: The responses of a pile-raft foundation under a moment when subjected to tunnelling. 


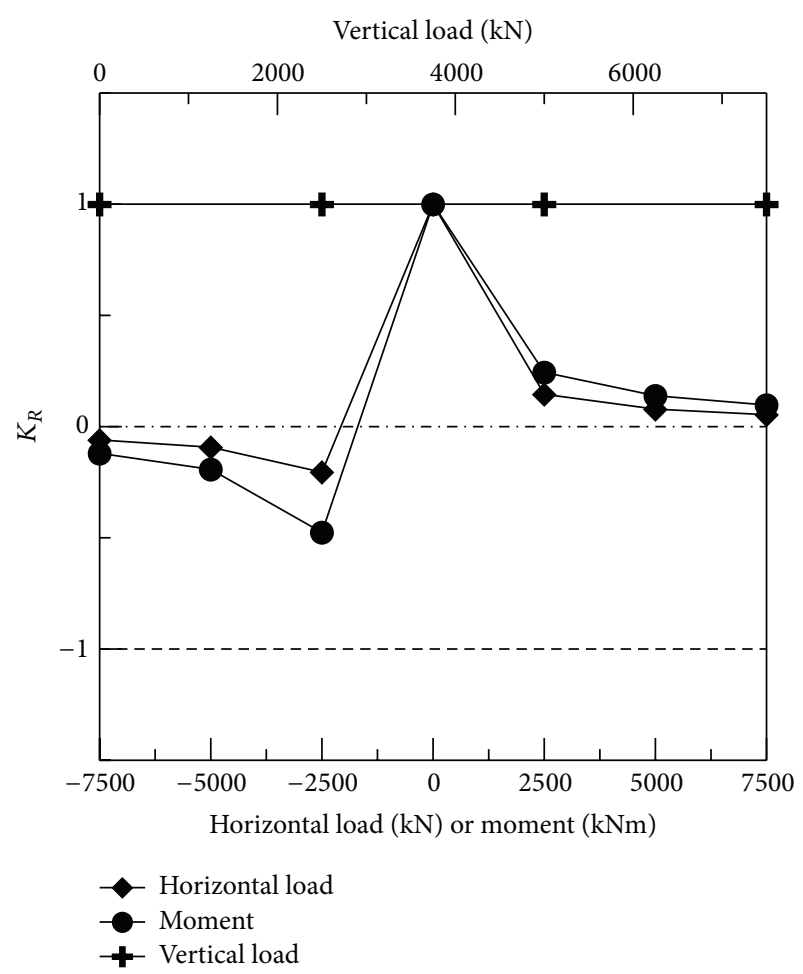

(a) Rotation

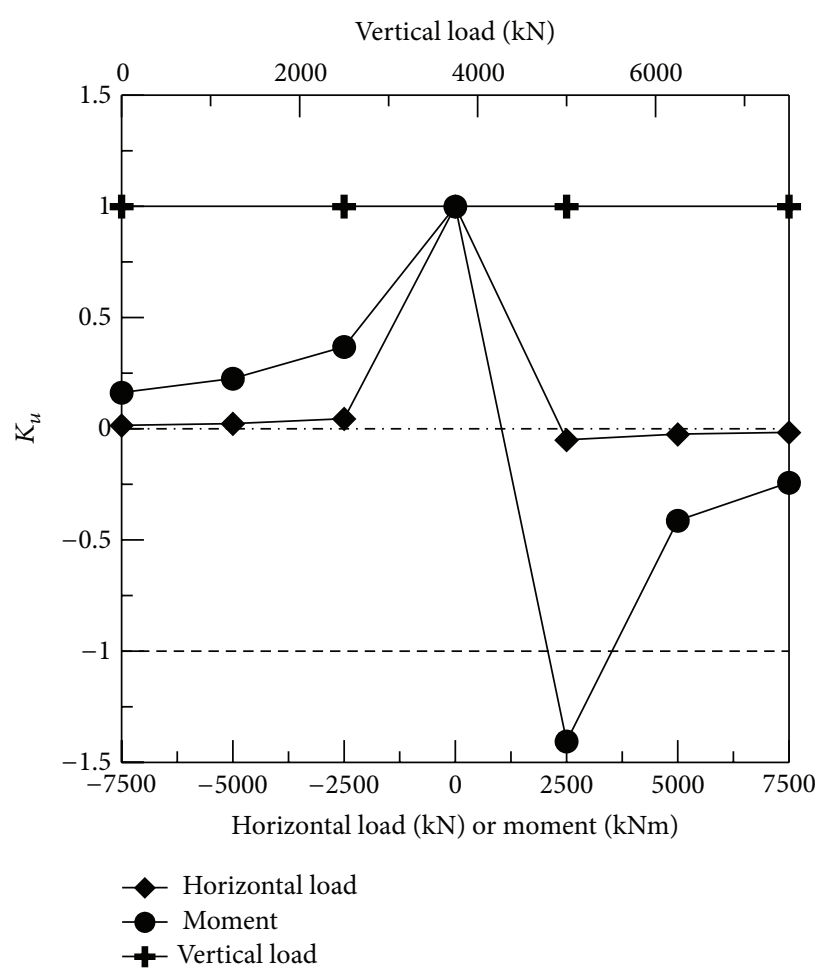

(b) Horizontal deformation

FIGURE 16: The influence of tunnelling on horizontal deformation and rotation.

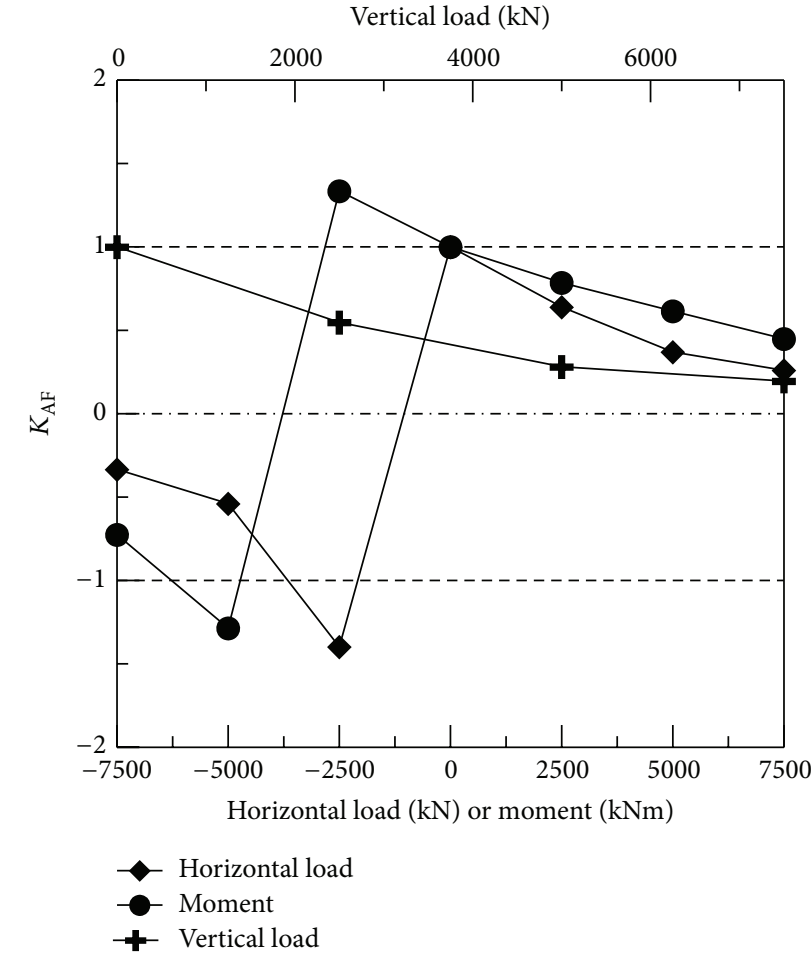

(a) Front pile

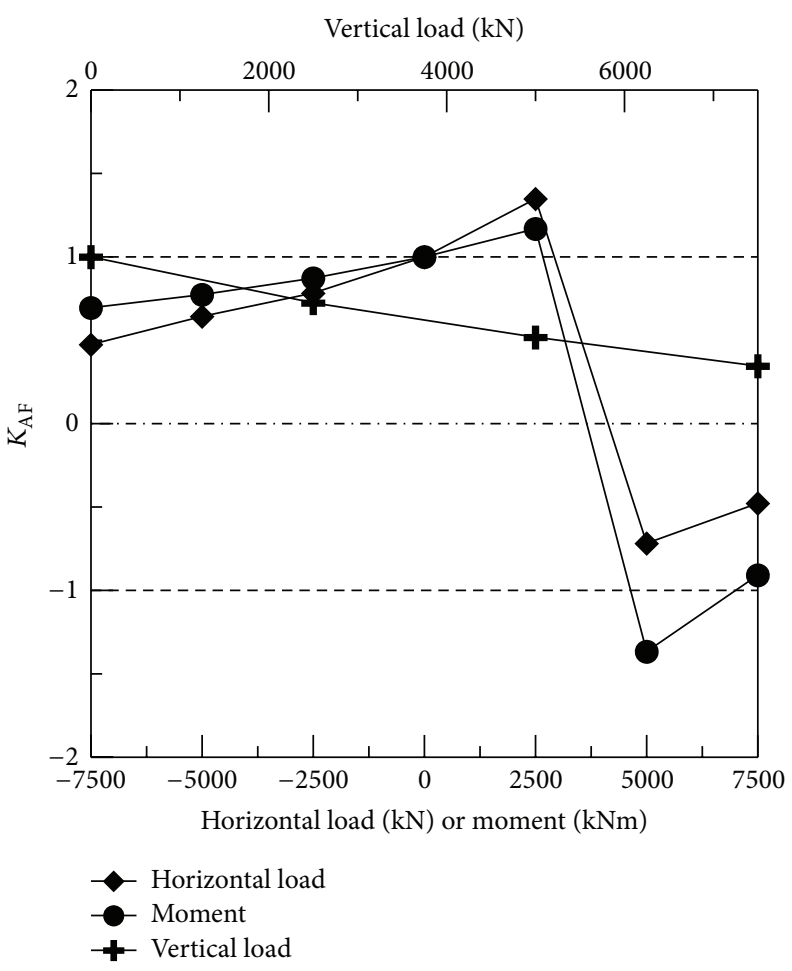

(b) Back pile

FIGURE 17: The influence of tunnelling on the axial force. 


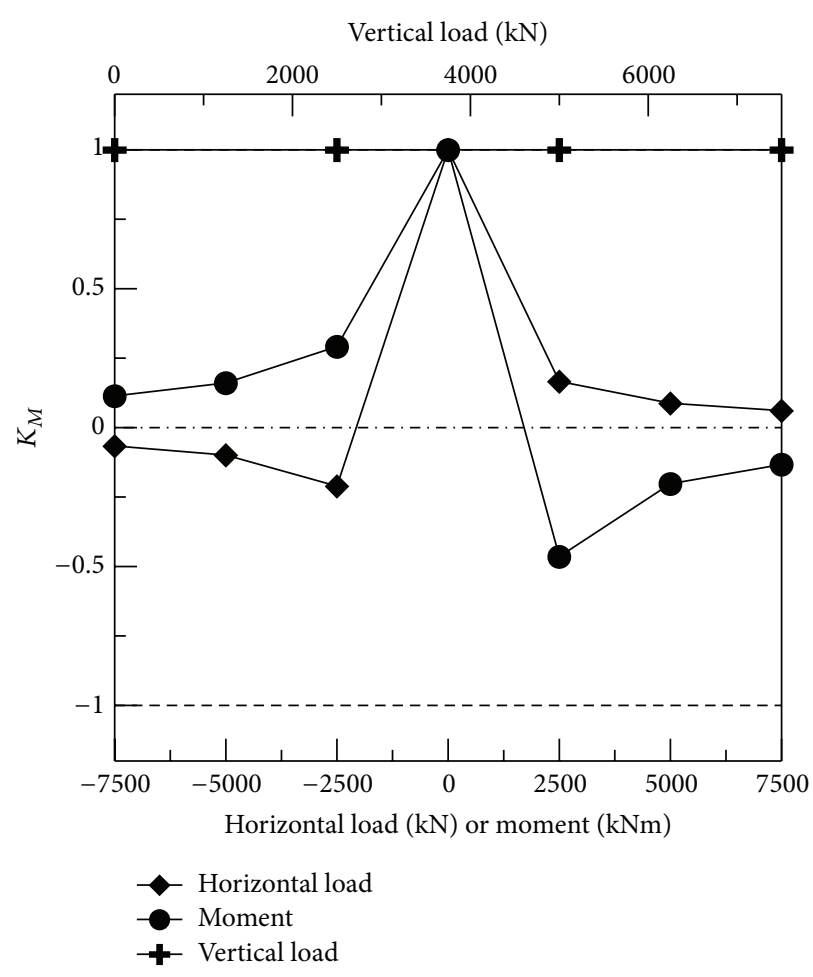

(a) Front pile

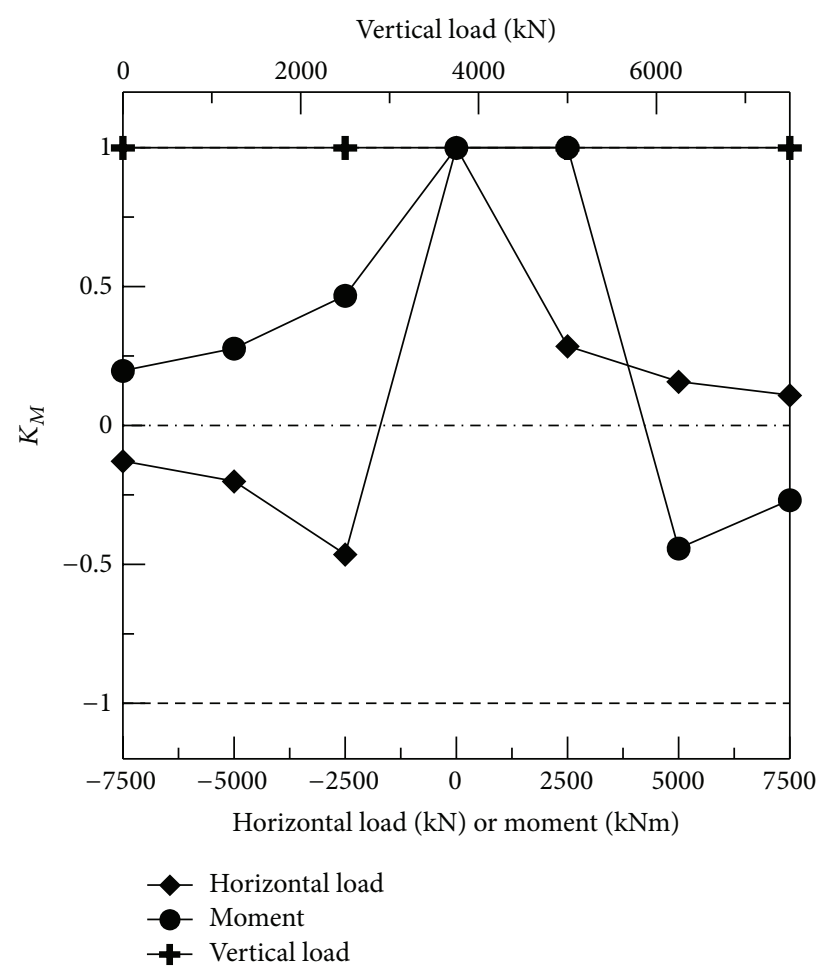

(b) Back pile

FIgURE 18: The influence of tunnelling on the moment.

finite-element methods, and other analytical procedures, an APPR program based on our modified two-stage method was proved to be a reasonable method for evaluating the responses of a multidirectionally loaded pile-raft foundation induced by tunnelling. A study of the influence factor showed that the smaller the absolute values of the loads became, the more necessary it was to consider the influence of tunnelling on loaded pile-raft foundations regardless of the direction of the active load.

\section{Conflict of Interests}

The authors declare that there is no conflict of interests regarding the publication of this paper.

\section{Acknowledgments}

The authors gratefully acknowledge the financial support from the National Science Fund of China (51208378) which was awarded to Linlong $\mathrm{Mu}$ (Tongji University, Shanghai) as Principal Investigator and the National Key Basic Research Program of China (2013CB036304).

\section{References}

[1] J. D. Morton and K. H. King, "Effects of tunnelling on the bearing capacity and settlement of piled foundations," in Proceedings of the 2nd International Symposium: Tunnelling '79, pp. 57-68, Institution of Mining and Metallurgy, London, UK, March 1979.
[2] N. Loganathan, H. G. Poulos, and D. P. Stewart, "Centrifuge model testing of tunnelling-induced ground and pile deformations," Geotechnique, vol. 50, no. 3, pp. 283-294, 2000.

[3] G. Neumann and M. Strembeck, "A scenario-driven role engineering process for functional RBAC roles," in Proceedings of the 7th ACM Symposium on Access Control Models and Technologies (SACMAT '02), pp. 33-42, ACM, Monterey, Calif, USA, June 2002.

[4] M. A. Meguid and J. Mattar, "Investigation of tunnel-soilpile interaction in cohesive soils," Journal of Geotechnical and Geoenvironmental Engineering, vol. 135, no. 7, pp. 973-979, 2009.

[5] H. Mroueh and I. Shahrour, "Three-dimensional finite element analysis of the interaction between tunneling and pile foundations," International Journal for Numerical and Analytical Methods in Geomechanics, vol. 26, no. 3, pp. 217-230, 2002.

[6] G. T. K. Lee and C. W. W. Ng, "Effects of advancing open face tunneling on an existing loaded pile," Journal of Geotechnical and Geoenvironmental Engineering, vol. 131, no. 2, pp. 193-201, 2005.

[7] C. Y. Cheng, G. R. Dasari, Y. K. Chow, and C. F. Leung, "Finite element analysis of tunnel-soil-pile interaction using displacement controlled model," Tunnelling and Underground Space Technology, vol. 22, no. 4, pp. 450-466, 2007.

[8] K. J. Xu and H. G. Poulos, "3-D elastic analysis of vertical piles subjected to passive loadings," Computers and Geotechnics, vol. 28, no. 5, pp. 349-375, 2001.

[9] N. Loganathan, H. G. Poulos, and K. J. Xu, "Ground and pilegroup responses due to tunnelling," Soils and Foundations, vol. 41, no. 1, pp. 57-67, 2001.

[10] P. Kitiyodom, T. Matsumoto, and K. Kawaguchi, "Analysis of piled raft foundation subjected to ground movement induced 
by tunnelling," in Proceedings of the 15th Southeast Asia Geotechnical Conference, pp. 183-188, Bangkok, Thailand, 2004.

[11] P. Kitiyodom, T. Matsumoto, and K. Kawaguchi, "A simplified analysis method for piled raft foundations subjected to ground movements induced by tunnelling," International Journal for Numerical and Analytical Methods in Geomechanics, vol. 29, no. 15, pp. 1485-1507, 2005.

[12] M. Huang, C. Zhang, and Z. Li, "A simplified analysis method for the influence of tunneling on grouped piles," Tunnelling and Underground Space Technology, vol. 24, no. 4, pp. 410-422, 2009.

[13] L. Mu, M. Huang, and R. J. Finno, "Tunnelling effects on lateral behavior of pile rafts in layered soil," Tunnelling and Underground Space Technology, vol. 28, no. 1, pp. 192-201, 2012.

[14] M. Huang and L. Mu, "Vertical response of pile raft foundations subjected to tunneling-induced ground movements in layered soil," International Journal for Numerical and Analytical Methods in Geomechanics, vol. 36, no. 8, pp. 977-1001, 2012.

[15] M. T. Davisson and K. E. Robinson, "Bending and buckling of partially embedded piles," in Proceedings of 6th International Conference on Soil Mechanics and Foundation Engineering, pp. 243-246, Montreal, Canada, 1965.

[16] B. F. Goryunov, "Analysis of piles subjected to the combined action of vertical and horizontal loads (discussion)," Soil Mechanics and Foundation Engineering, vol. 10, no. 1, pp. 10-13, 1973.

[17] G. Ramaswamy, Flexural behavior of axially and laterally loaded individual piles and group of piles [Ph.D. thesis], Indian Institute of Science, 1974.

[18] C. Anagnostopoulos and M. Georgiadis, "Interaction of axial and lateral pile responses," Journal of Geotechnical Engineering, vol. 119, no. 4, pp. 793-798, 1993.

[19] C.-J. Lee and K.-H. Chiang, "Responses of single piles to tunneling-induced soil movements in sandy ground," Canadian Geotechnical Journal, vol. 44, no. 10, pp. 1224-1241, 2007.

[20] R.-J. Zhang, J.-J. Zheng, L.-M. Zhang, and H.-F. Pu, "An analysis method for the influence of tunneling on adjacent loaded pile groups with rigid elevated caps," International Journal for Numerical and Analytical Methods in Geomechanics, vol. 35, no. 18, pp. 1949-1971, 2011.

[21] L. Mu, M. Huang, and K. Lian, "Analysis of pile-raft foundations under complex loads in layered soils," International Journal for Numerical and Analytical Methods in Geomechanics, vol. 38, no. 3, pp. 256-280, 2014.

[22] C. Sagaseta, "Analysis of undrained soil deformation due to ground loss," Géotechnique, vol. 37, no. 3, pp. 301-320, 1987.

[23] A. Verruijt and J. R. Booker, "Surface settlements due to deformation of a tunnel in an elastic half plane," Géotechnique, vol. 46, no. 4, pp. 753-756, 1996.

[24] M. J. Gunn, "The predictive of surface settlement profiles due to tunnelling," in Proceedings of the Wroth Memorial Symposium, pp. 304-316, Oxford, UK, 1993.

[25] R. B. Peck, "Deep excavations and tunnelling in soft ground," in Proceedings of the 7th International Conference on Soil Mechanics and Foundation Engineering (SMFE '69), pp. 225290, Mexico City, Mexico, 1969.

[26] R. J. Mair, "Ground movements around shallow tunnels in soft clay," in Proceedings of the 10th ICSMFE, pp. 323-328, Stockholm, Sweden, 1993.

[27] N. Loganathan and H. G. Poulos, "Analytical prediction for tunneling-induced ground movements in clays," Journal of Geotechnical and Geoenvironmental Engineering, vol. 124, no. 9, pp. 846-856, 1998.
[28] Y. K. Chow and C. I. Teh, "A theoretical study of pile heave," Géotechnique, vol. 40, no. 1, pp. 1-14, 1990.

[29] P. Kitiyodom and T. Matsumoto, "A simplified analysis method for piled raft foundations in non-homogeneous soils," International Journal for Numerical and Analytical Methods in Geomechanics, vol. 27, no. 2, pp. 85-109, 2003. 


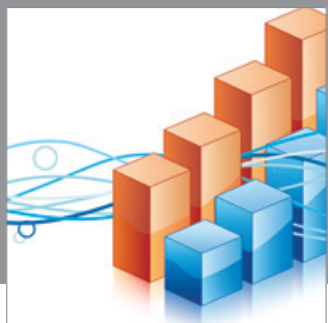

Advances in

Operations Research

mansans

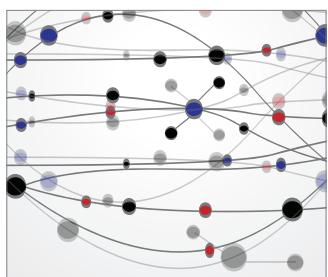

The Scientific World Journal
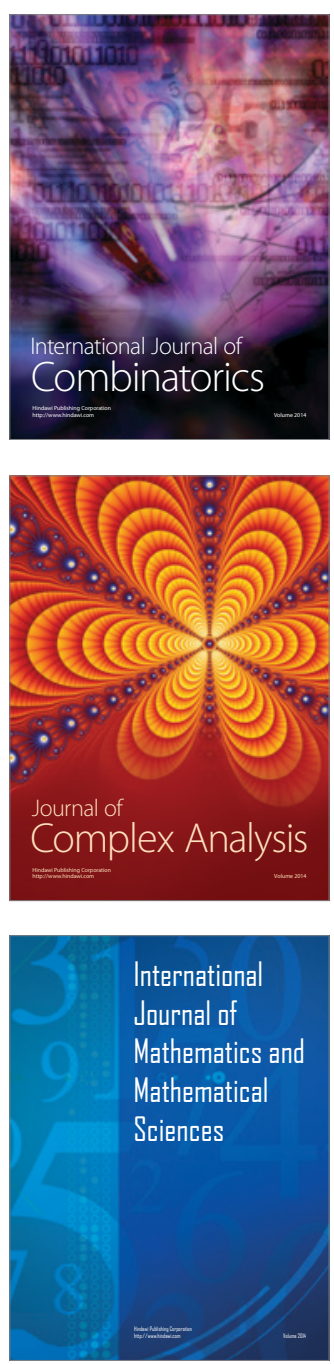
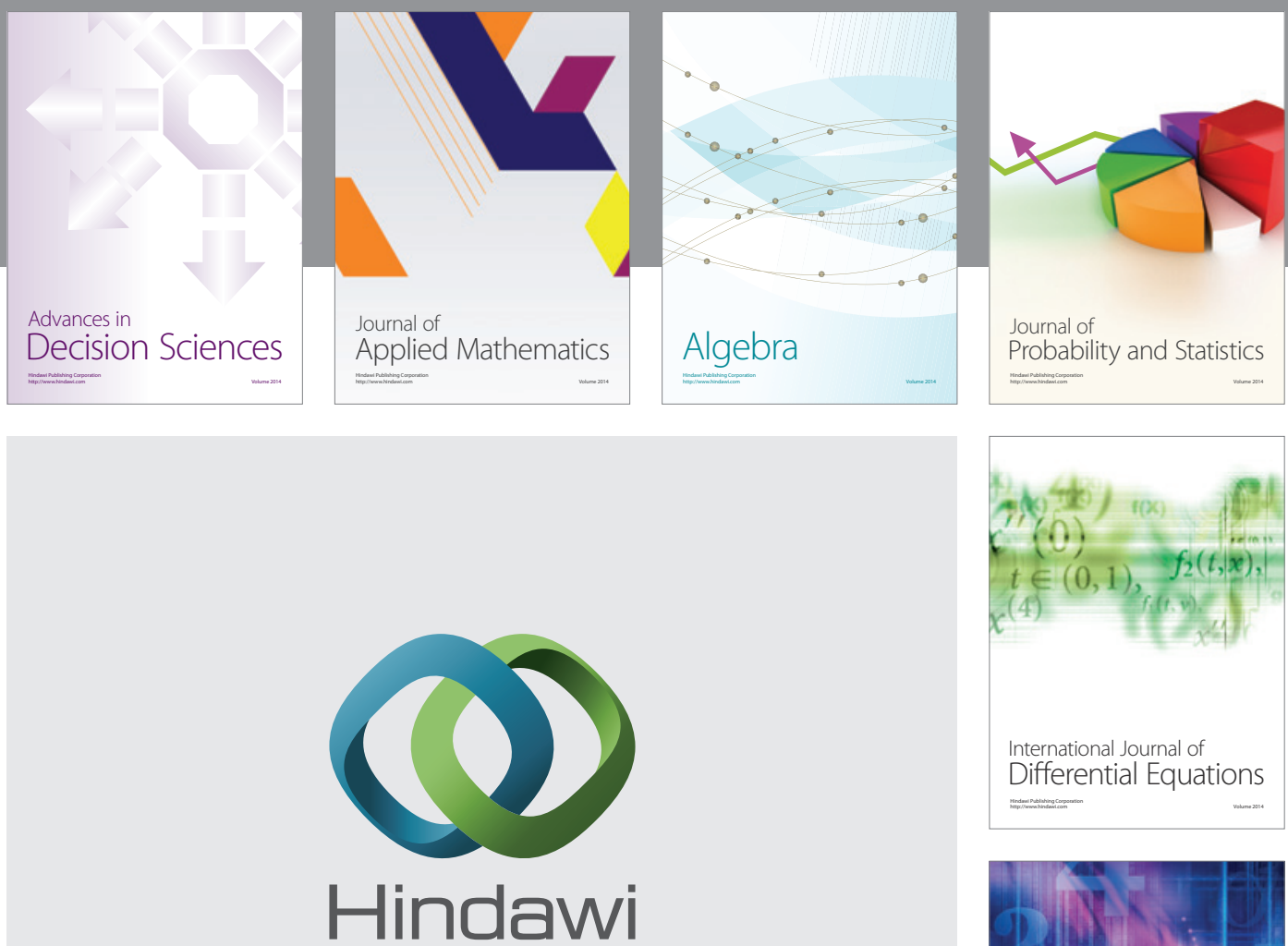

Submit your manuscripts at http://www.hindawi.com
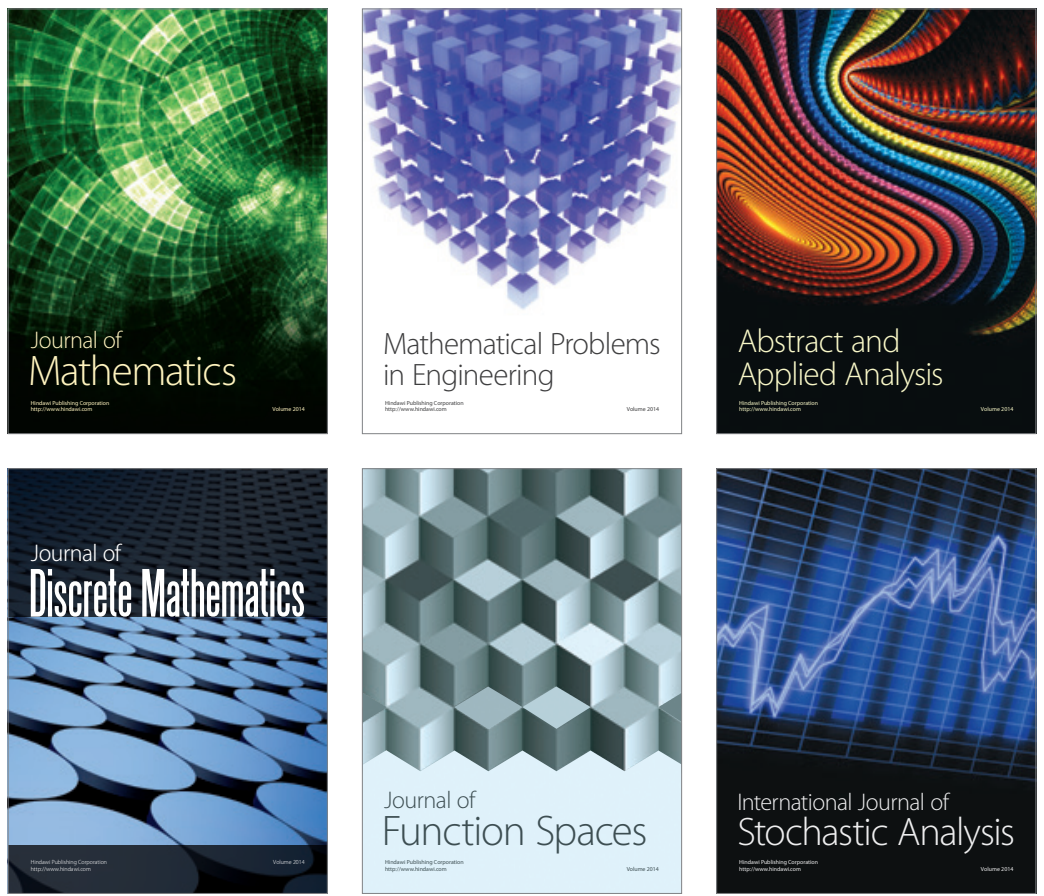

Journal of

Function Spaces

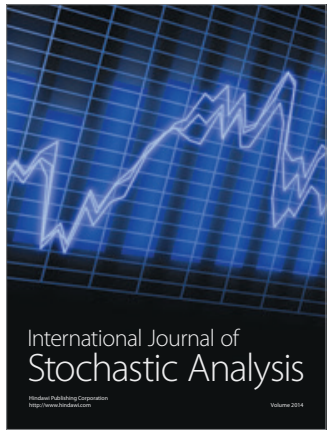

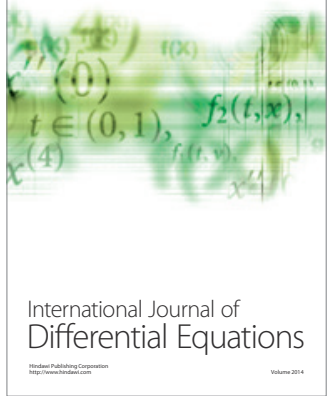
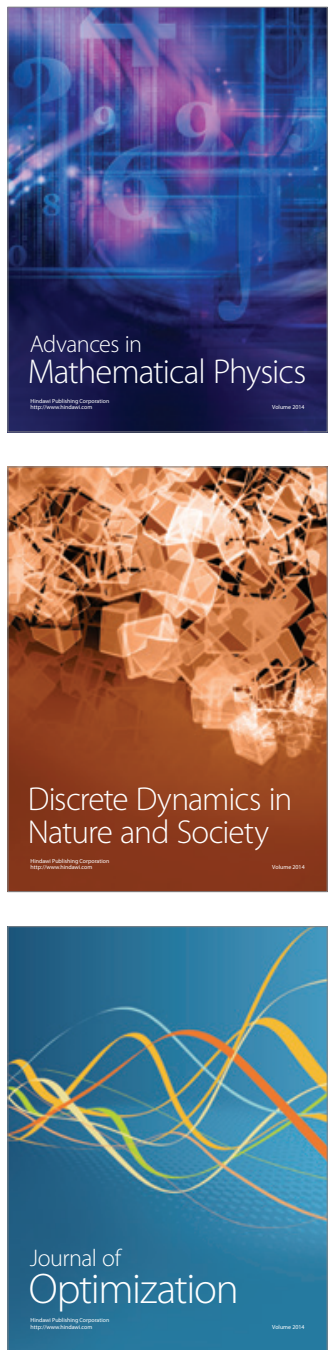